\title{
HIGH-FREQUENCY GLOBAL WAVEFIELDS FOR LOCAL 3D STRUCTURES BY WAVEFIELD INJECTION AND EXTRAPOLATION
}

\author{
PREPRINT
}

\author{
Marta Pienkowska $^{1,2}$, Vadim Monteiller ${ }^{3}$, and Tarje Nissen-Meyer ${ }^{1}$ \\ ${ }^{1}$ Department of Earth Sciences, University of Oxford, Oxford, United Kingdom \\ ${ }^{2}$ Department of Earth Sciences, ETH Zurich, Zurich, Switzerland \\ ${ }^{3}$ Laboratoire de Mécanique et d'Acoustique, LMA - UMR 7031 AMU - CNRS - Centrale Marseille
}

\begin{abstract}
Earth structure is multiscale, and seismology remains the primary means of deciphering signatures from small structures over large distances. To enable this at the highest resolution, we present a flexible injection and extrapolation type hybrid framework that couples wavefields from a precomputed global database of accurate Green's functions with a local three dimensional (3-D) method of choice (e.g. a spectral element of a finite difference solver). The interface allows to embed a full 3-D domain in a spherically symmetric Earth model, tackling large-scale wave propagation with focus on localized heterogeneous complex structures. Thanks to reasonable computational costs (10k CPU hours) and storage requirements (a few TB for $1 \mathrm{~Hz}$ waveforms) of databases of global Green's functions, the method provides coupling of 3-D wavefields that can reach the highest observable body-wave frequencies in the $1-4 \mathrm{~Hz}$ range. The framework is highly flexible and adaptable; alterations in source properties (radiation patterns, source-time function), in the source-receiver geometry, and in local domain dimensions and location can be introduced without re-running the global simulation. The once-and-for-all database approach reduces the overall computational cost by a factor of 5,000-100,000 relative to a full 3-D run, provided that the local domain is of the order of tens of wavelengths in size. In this paper we present the details of the method and its implementation, show benchmarks with a 3-D spectral-element solver, discuss its setup-dependent performance, and explore possible wave-propagation applications.
\end{abstract}

Keywords computational seismology $\cdot$ wave propagation $\cdot$ wave scattering and diffraction $\cdot$ numerical solutions $\cdot$ body waves

\section{INTRODUCTION}

The past few decades have seen a significant improvement in our capacity to image the Earth's interior. Rapid hardware and software developments, along with increasing and more accessible high-quality broadband data, have driven progress in our attempts to infer the Earth's structure and dynamics [5,20] However, even though we understand wave propagation and its numerical solution, it remains computationally prohibitive to generate high frequency synthetic waves in a realistic, multiscale, heterogeneous Earth - a challenge especially relevant when considering global scale problems.

Theoretically, with full three-dimensional (3-D) numerical solvers at hand, such as finite difference [29, 43], discontinuous Galerkin [19] and spectral element [14, 32, 33] methods, we can tackle media of various geometries and desired complexities. The computational cost of those methods, however, scales with frequency to the $4^{\text {th }}$ power (three dimensions in space, one in time), decidedly restricting their applicability to global scale problems for the observable frequency band: at present, frequencies around $1 \mathrm{~Hz}$ can be considered the practical limit for a few full 3-D global simulations on large clusters [35, 60]. Since iterative inverse problems or systematic studies of synthetic waveforms require large numbers of simulations, in practice such full 3-D methods remain unfeasible at high frequencies especially at the global scale where waves travel over thousands of wavelengths in distance. Given the dependence of computational cost on the frequency, this will remain a bottleneck for some time even on the largest future infrastructures.
As a result, to compute high-frequency body waves we often rely on methods that include the full physics of wave propagation but reduce media complexity by assuming one-dimensional (1-D) spherically symmetric models, such as DSM [31], GEMINI [25], Yspec [2], or AxiSEM [48]. Such structural simplification facilitates wave propagation for realistic Earth models and provides a compromise between broad frequency ranges and computational costs, allowing for direct comparisons of synthetics with observed waveform data [48]. Leng et al. [36], however, show in a synthetic study that 3-D scatterers of a couple of wavelengths in size have a recognisable impact on high-frequency global wave propagation, an effect that is currently largely ignored. Along with insufficient data coverage, such structural modelling assumptions resulted in global tomographic models that are in reasonable agreement for large-scale structures [4, 5], but diverge at smaller length scales. Improving seismic Earth models and our understanding of waveforms must therefore incorporate numerical methods that not only capture the relevant physics and provide a high-frequency resolution, but also account in a computationally feasible manner for the complexity that affects global wave propagation in 3-D.

More recently, AxiSEM3D [35] has drastically improved our capability to generate high-frequency synthetic wavefields on a global scale. Leng et al. [35] proposed to exploit the smoothness of global wavefields to reduce computational costs and characterized the azimuthal dependence of 3-D wavefields in terms of Fourier series. Later, van Driel et al. [64] have adopted the smoothness idea to generate wavefield-adapted three-dimensional meshes. The efficiency of both methods is 
therefore model dependent, and increasing model complexity increases numerical requirements. Even though the methods can be applied to highly heterogeneous structures and provide a significant speed-up relative to full 3-D solvers and meshes, they nonetheless remain prohibitive for routine use at resolutions above $1 \mathrm{~Hz}$.

Hybrid methods that couple two solvers can bridge that gap between high structural complexity and computational cost. Such techniques represent the bulk 'background' medium as a sparse or smooth structure that accounts for source and path effects, and honour complexities in the model to resolve local scattering in areas of interest. The wavefields are coupled on the boundary between the subdomains, effectively embedding smaller-scale structures in the larger model. Each method provides accurate solutions in its domain of validity, while the reduced complexity (e.g. 1-D model) or approximate physics (e.g. ray theory or the frequency-wavenumber (FK) method) in the background medium decrease the overall computational cost of the problem.

A large proportion of coupling methods proposed to date in seismic wave propagation, often referred to as wavefield injection or domain reduction methods, stem from the work by Alterman and Karal [3] addressing the source singularity problem in finite difference schemes. Such methods are effectively a two-step procedure with no dynamic exchange of information between the solvers. They have been evolving over the years as numerical solvers themselves evolved, striving to efficiently obtain ever better wavefield estimates for both the incident background field and the local scattered field. The vast majority offers to couple the local domain directly under the receiver arrays on the surface [e.g., 8, 9, 15, 41, 42, 44, 49, 50, 54, 55, 59, 67, 74], and only a few of the injection methods are suited for full 3-D global rather than regional applications. Chevrot et al. [15] presented the first implementation of injection of teleseismic body waves into a 3-D spectral-element solver, though the local 3-D domain was embedded in a homogeneous background medium and only incoming planar wave fronts were considered. Later, Monteiller et al. [44] coupled 1-D DSM with Specfem3D Cartesian, Tong et al. [59] coupled 1-D frequency-wavenumber (FK) wavefields with Specfem3D Cartesian, Masson and Romanowicz [39, 40] coupled Specfem3D Globe with regSEM [18], and Beller et al. $[6,7]$ coupled 1-D AxiSEM with Specfem3D Cartesian. To the best of the authors' knowledge, only three global-scale 3-D methods go beyond wavefield injection and include domains away from the receivers - around the source [40,70], or at depth $[38,39,40]$ - that extrapolate local wavefields to stations at distance. Masson and Romanowicz [39, 40], however, focus on fully 3-D structures in both the background and the local domains, and the high computational requirements of the global background 3-D model restrict the applicability of their method for the uppermost frequencies observed teleseismically. Lin et al. [38], on the other hand, propose a 2-D conceptual study of the full workflow for local spectral-element methods, with a possible extension to 3-D. Other two-way coupling methods have also been proposed to account for higher order long range scattering between the two domains [11, 12, 13, 26, 65, 68], but dynamic boundary conditions on the interface between the subdomains require a significant computational effort and remain expensive for large numbers of high-frequency simulations.
Offering advantages not provided by either of the methods on their own, hybrid methods have recently been used to study the effects of Moho and surface topography on teleseismic wavefields [44], to carry out parametric studies of inversion methods $[7,73]$, and to locally improve the resolution of tomographic models $[16,39]$. Although a coupling of two solvers does not provide the ultimate solution to high-frequency simulations on global scale, it is well-suited for a wide range of applications and can bring us closer to understanding the multi-scale Earth structure and the imprints of small heterogeneities - around sources, receivers, or at depth - on the global wavefield.

The methodology put forward in this paper fills the gap for a global scale hybrid coupling that includes the full physics, targets the highest observed body-wave frequencies and caters for both wavefield injection and extrapolation. It provides an unprecedented level of flexibility, with a framework built around 1-D spherically symmetric Earth models that allows for coupling with an arbitrary local 3-D solver of choice. The framework employs Instaseis [63, www.instaseis.net], a tool that allows a near-instantaneous extraction of global Green's functions from pre-computed AxiSEM [48, www.axisem.info] databases. Such databases act as a once-and-for-all solution to wave propagation in spherically symmetric background models, leaving the flexibility with respect to parameter alterations, such as modifications in source properties (radiation patterns, source-time function), in the source-receiver geometry, and in local domain dimensions and location (including regions around the source or receivers, and at depth) to subsequent hybrid applications without the need of re-running the global simulation. Thanks to reasonable computational costs (about 10,000 CPU hours for a re-usable database) and storage requirements (a few TB for global $1 \mathrm{~Hz}$ waveforms) of the Instaseis databases, the hybrid method can reach the highest observable body-wave frequencies in global seismology and opens numerical simulations to new parameter regimes.

The paper is structured as follows. In Section 2 we first review the principles of wavefield injection and of wavefield extrapolation (via the Helmholz-Kirchhoff integral in the representation theorem) which form the basis of the one-way methods and connect the solution in two subdomains. We then look in Section 3 at the specificities of the implementation of the coupling with global AxiSEM wavefields and Instaseis databases. In Section 4 we validate the framework with two very different local solvers and discuss the key parameters defining the efficiency and performance of hybrid methods. We also compare the hybrid runs to full global runs, with the coupled framework performing up to 100,000 times faster than 3-D global solvers. In Section we explore a range of possible applications, and in Section 6 we discuss the method with its limitations, further developments and extensions.

\section{THEORY}

Hybrid methods decompose the computational domain into subdomains - often referred to as the background and local domains - in order to exploit advantages of individual solvers and increase algorithmic efficiency. Wavefield injection caters for cases when the source is in the background domain, and the wavefield needs to be imposed in the local domain that is embedded therein. The Helmholz-Kirchhoff integrals, on the other hand, allow to ex- 


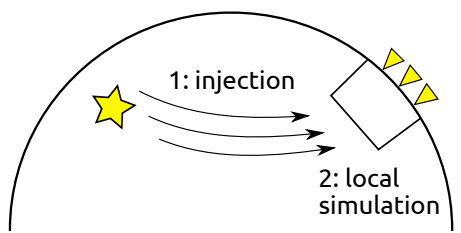

(a)

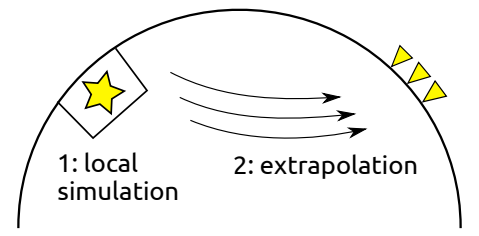

(b)

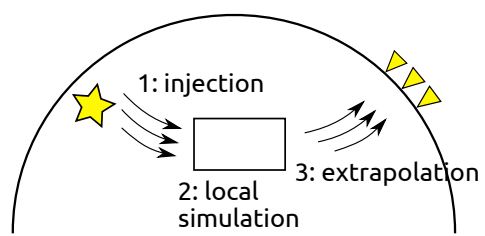

(c)

Figure 1: Wavefield injection and wavefield extrapolation methods that couple solutions in two domains provide a framework for three set-ups: (a) With the local domain under the receivers via wavefield injection. The wavefield is propagated through the background (1) and then injected into the local domain (2). (b) With the local domain around the source region via wavefield extrapolation. The source is simulated in the local domain (1) and the wavefield is then extrapolated to the receivers at distance (2). (c) With the local domain at depth, via a combination of injection and extrapolation. The wavefield is propagated into the local domain (1), interactions at depth are accounted for locally (2), and finally repropagated to the receivers at distance (3).

trapolate the wavefield from the local domain around the source region to receivers at distance in the background medium. Together the two provide a framework for three distinct set-ups: with the local domain around the receiver (injection, Fig. 1a), around the source (extrapolation, Fig. 1b), and at depth (both injection and extrapolation, Fig. 1c).

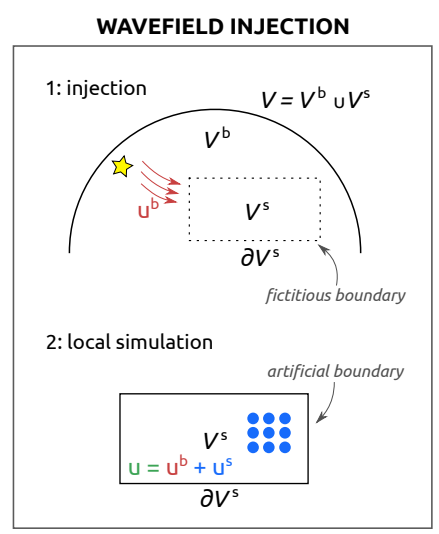

(a)

\section{WAVEFIELD EXTRAPOLATION}

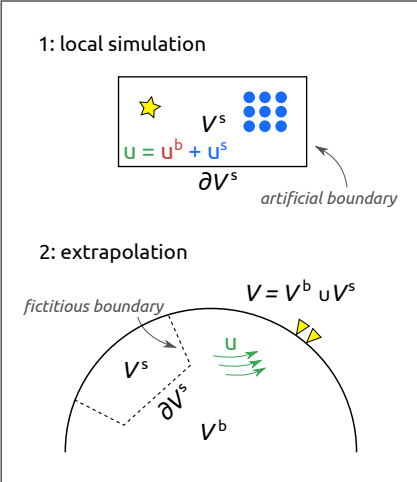

(b)
Figure 2: Definition of domains involved in wavefield injection and extrapolation. Background volume $V=V^{b} \cup V^{s}$ is subdivided by a fictitious interface $\partial V^{s}$, where subdomain $V^{s}$ defines the truncated volume represented in a local solver. Modifying the structure in $V^{s}$ in the local solver, hybrid coupling accounts for local interactions and the wavefield $\mathbf{u}(\mathbf{x}, t)$ in $V^{s}$ is a linear combination of the background wavefield $\mathbf{u}^{b}(\mathbf{x}, t)$ and the scattered wavefield $\mathbf{u}^{s}(\mathbf{x}, t)$. Note that the structure of the coupling interface $\partial V^{s}$ itself must remain unchanged relative to its representation in the background volume $V$. With $V^{s}$ the same as its representation in the background volume $V$ there is no residual scattered wavefield relative to the background model and $\mathbf{u}^{s}(\mathbf{x}, t)=0$, i.e. hybrid coupling reproduces the original wavefield $\mathbf{u}^{b}(\mathbf{x}, t)$. (a) For injection, subdomain $V^{b}$ contains the seismic source. Background wavefield $\mathbf{u}^{b}(\mathbf{x}, t)$ is recorded on the fictitious boundary $\partial V^{s}$. The volume $V^{s}$ is represented in a local solver and the wavefield $\mathbf{u}^{b}(\mathbf{x}, t)$ is imposed on the boundary. (b) The volume $V^{s}$ is represented in a local solver that includes the seismic source, and the local wavefields are recorded on $\partial V^{s}$. The fictitious boundary $\partial V^{s}$ is used to repropagate the wavefield $\mathbf{u}(\mathbf{x}, t)$ from the local solver to receivers at distance.

\subsection{Wavefield injection}

Wavefield injection introduces an incident wavefield generated by a source in a background medium into a local domain embedded in that medium. The source and the majority of the path effects are accounted for in the background domain and are carried over to a regional solver via the local boundary: either via imposing appropriate boundary conditions that drive the local simulation [54] or by introducing a distribution of body forces on the local boundary [41]. The method therefore consists of two steps. First, the interactions in the background domain are simulated and recorded on a fictitious boundary of an area of interest. Next, that area is represented in a local solver, with the boundary introducing the background wavefield.

Let $V$ be a linearly elastic background model subdivided by a fictitious interface $\partial V^{s}$ such that $V^{s}$ is the region of the local truncated volume embedded in $V$, and $V^{b}$ the part of the domain including the seismic source, i.e. $V=V^{b} \cup V^{s}$ (Fig. 2a). The domain $V^{s}$, along with its bounding interface $\partial V^{s}$, has two representations, one in each solver:

- In the global solver, $V=V^{b} \cup V^{s}$ is represented, and $\partial V^{s}$ is a fictitious, non-physical boundary that surrounds the region of interest $V^{s}$ within the global domain $V . \partial V^{s}$ serves only as a coupling interface.

- In the local solver only $V^{s}$ is represented, while $\partial V^{s}$ is an artificial, non-physical boundary that serves as a coupling interface and requires absorbing boundary conditions.

In the first step, the background wavefield $\mathbf{u}^{b}(\mathbf{x}, t)$ is recorded on $\partial V^{s}$ within the global domain $V$. In the second step, the volume $V^{s}$ is represented in a different solver and the wavefield $\mathbf{u}^{b}(\mathbf{x}, t)$ is imposed on the boundary $\partial V^{s}$. If the model in the local domain remains identical to the representation of $V^{s}$ in the background model, there is no residual scattered wavefield relative to the background model and the coupling should reproduce the original background wavefields in $V^{s}$, i.e. $\mathbf{u}^{s}(\mathbf{x}, t)=0$ and $\mathbf{u}(\mathbf{x}, t)=\mathbf{u}^{b}(\mathbf{x}, t)$. When the model in $V^{s}$ is modified to account for complex local structures, the total wavefield $\mathbf{u}(\mathbf{x}, t)$ in the local solver becomes $\mathbf{u}(\mathbf{x}, t)=\mathbf{u}^{b}(\mathbf{x}, t)+\mathbf{u}^{s}(\mathbf{x}, t)$, where $\mathbf{u}^{s}(\mathbf{x}, t) \neq 0$ represents local scattering. In what follows, we discuss two ways of imposing the background wavefield on the boundary of the local domain to drive the local simulation. 


\subsubsection{Boundary condition}

The background wavefield $\mathbf{u}^{b}(\mathbf{x}, t)$ can be imposed via a Dirichlet condition on the boundary of the local domain. Let's first consider the total displacement wavefield $\mathbf{u}(\mathbf{x}, t)$ that is a solution to the elastodynamic equations of motion

$$
\begin{cases}\rho \ddot{u}_{n}-\partial_{j} \sigma_{n j}=f_{n} & \text { in } V^{s} \cup V^{b} \\ \sigma_{n j}=C_{n j k l} \partial_{k} u_{l} & \text { in } V^{b} \\ \sigma_{n j}=\mathcal{H}[\mathbf{u}(\mathbf{x}, t)] & \text { in } V^{s} \\ \sigma_{n j} n_{n}=0 & \text { on } \partial V \\ u_{n}^{+}=u_{n}^{-} & \text {on } \partial V^{s} \\ \sigma_{n j}^{+} n_{n}=\sigma_{n j}^{-} n_{n} & \text { on } \partial V^{s}\end{cases}
$$

where $\mathcal{H}[\mathbf{u}(\mathbf{x}, t)]$ represents a general constitutive relation in $V^{s}$, and where we assume $\partial V$ to be the Earth's free surface, as we are primarily concerned with global scale applications. The plus and minus superscripts denote limits from $V^{b}$ and $V^{s}$, respectively, to warrant continuity of displacement and traction across any internal boundaries. The wavefields and constitutive relations are required to be continuous on the boundary $\partial V^{s}$. While the background model is (visco)elastic in $V^{b}$, the general relation $\mathcal{H}[\mathbf{u}(\mathbf{x}, t)]$ in $V^{s}$ does not require an elastic behaviour in $V^{s}$ Hence $V^{s}$ can encompass, for example, non-linear interactions or kinematic ruptures provided that $\partial V^{s}$ remains (visco)elastic. Given the linearly elastic model in $V^{b}$ and on $\partial V^{s}$, we can define hybrid variables $\mathbf{u}^{h}(\mathbf{x}, t), \sigma^{h}(\mathbf{x}, t)$ such that

$$
\mathbf{u}^{h}(\mathbf{x}, t)=\left\{\begin{array}{l}
\mathbf{u}(\mathbf{x}, t) \\
\mathbf{u}(\mathbf{x}, t)-\mathbf{u}^{b}(\mathbf{x}, t)
\end{array}= \begin{cases}\mathbf{u}(\mathbf{x}, t) & \text { in } V^{s} \\
\mathbf{u}^{s}(\mathbf{x}, t) & \text { in } V^{b}\end{cases}\right.
$$

(and similarly for $\boldsymbol{\sigma}^{h}(\mathbf{x}, t)$ ) where $\mathbf{u}^{b}(\mathbf{x}, t)$ is the background wavefield computed in the first step, $\mathbf{u}^{s}(\mathbf{x}, t)$ is the scattered wavefield due to 3-D model heterogeneities in $V^{s}$, and $\mathbf{u}(\mathbf{x}, t)$ is the total wavefield that solves equation (1). We then want to find the hybrid field $\mathbf{u}^{h}(\mathbf{x}, t)$ such that

$$
\begin{cases}\rho \ddot{u}_{n}^{h}-\partial_{j} \sigma_{n j}^{h}=0 & \text { in } V^{s} \cup V^{b} \\ \sigma_{n j}^{h}=C_{n j k l} \partial_{k} u_{l}^{h} & \text { in } V^{b} \\ \sigma_{n j}^{h}=\mathcal{H}\left[\mathbf{u}^{h}(\mathbf{x}, t)\right] & \text { in } V^{s} \\ \sigma_{n j}^{h} n_{n}=0 & \text { on } \partial V \\ u_{n}^{h^{+}}=u_{n}^{h^{-}}-u_{n}^{b} & \text { on } \partial V^{s} \\ \sigma_{n j}^{h+} n_{n}=\sigma_{n j}^{h} n_{n}-\sigma_{n j}^{b} n_{n} & \text { on } \partial V^{s}\end{cases}
$$

In the new problem, unlike in equation (1), the information about the excitation is given by a Dirichlet condition on $\partial V^{s}$ that imposes a discontinuity in the wavefield. This result could be extended to problems with a non-linear constitutive relation in $V^{b}$ as long as $\mathbf{u}^{h}(\mathbf{x}, t)$ remains a linear perturbation around $\mathbf{u}^{b}(\mathbf{x}, t)$ on $\partial V^{s}$.

Posing this problem as above, we can see that the boundary $\partial V^{s}$ not only generates the complete field $\mathbf{u}(\mathbf{x}, t)$ in $V^{s}$, but also the scattered field $\mathbf{u}^{s}(\mathbf{x}, t)$ propagating out of $\partial V^{s}$. In practice, however, the second computation happens only in the truncated subdomain $V^{s}$ with absorbing boundary conditions applied on $\partial V^{s}$ to the outgoing field $\mathbf{u}^{s}(\mathbf{x}, t)$. The background wavefield $\mathbf{u}^{b}(\mathbf{x}, t)$ can be computed with any numerical or analytic method provided that the wavefield can be interpolated to the grid points of the local solver.

\subsubsection{Distribution of point sources}

Wavefield injection can also be implemented via a discretisation of the surface integrals in the representation theorem into multiple point sources. Applying the representation theorem to the background wavefield $\mathbf{u}^{b}(\mathbf{x}, t)$ in $V^{b}$ we have:

$$
\begin{aligned}
& u_{i}^{b}(\mathbf{x}, t)= \\
& \quad \int_{-\infty}^{\infty} d \tau \int_{V^{b}} f_{i}^{b}(\boldsymbol{\xi}, \tau) G_{i n}(\boldsymbol{\xi}, t-\tau ; \mathbf{x}, 0) \mathrm{d} V^{b} \\
& +\int_{-\infty}^{\infty} d \tau \int_{\partial V^{s}} n_{j} C_{i j k l} \partial_{k} u_{l}^{b}(\boldsymbol{\xi}, \tau) G_{i n}(\boldsymbol{\xi}, t-\tau ; \mathbf{x}, 0) \mathrm{d}\left(\partial V^{s}\right) \\
& -\int_{-\infty}^{\infty} d \tau \int_{\partial V^{s}} n_{j} C_{i j k l} u_{i}^{b}(\boldsymbol{\xi}, \tau) \partial_{l} G_{k n}(\boldsymbol{\xi}, t-\tau ; \mathbf{x}, 0) \mathrm{d}\left(\partial V^{s}\right)
\end{aligned}
$$

where $\mathbf{n}$ is the normal on $\partial V^{s}$ pointing outwards of $V^{b}$. Given a global background domain in our methodology $\partial V$ is assumed to be the Earth's stress-free surface, so that integrals over $\partial V$ vanish and only integrals over $\partial V^{s}$ remain in (4). Note that this represents the background wavefield in $V^{b}$ only.

Recall now that we aim to impose the background wavefield on the boundary of the local domain in order to represent (inject) that background wavefield in $V^{s}$. The wavefield $\mathbf{u}^{b}(\mathbf{x}, t)$ does not have sources in $V^{s}$, so $\mathbf{f}^{b}(\mathbf{x}, t)=0 \forall \mathbf{x} \in V^{s}$ and the volume integral vanishes. Thus, for $\mathbf{u}^{b}(\mathbf{x}, t)$ in $V^{s}$ we get

$$
\begin{aligned}
& u_{i}^{b}(\mathbf{x}, t)= \\
& \quad \int_{-\infty}^{\infty} d \tau \int_{\partial V^{s}}\left(n_{j}^{\prime}\right) C_{i j k l} \partial_{k} u_{l}^{b}(\boldsymbol{\xi}, \tau) G_{i n}(\boldsymbol{\xi}, t-\tau ; \mathbf{x}, 0) \mathrm{d}\left(\partial V^{s}\right) \\
& -\int_{-\infty}^{\infty} d \tau \int_{\partial V^{s}}\left(n_{j}^{\prime}\right) C_{i j k l} u_{i}^{b}(\xi, \tau) \partial_{l} G_{k n}(\boldsymbol{\xi}, t-\tau ; \mathbf{x}, 0) \mathrm{d}\left(\partial V^{s}\right),
\end{aligned}
$$

where $\mathbf{n}^{\prime}=-\mathbf{n}$ is the normal on $\partial V^{s}$ pointing outwards of $V^{s}$. This result can be rigorously derived by considering a surface-source type of problem of scattering by an arbitrary inhomogeneous object [24]. Approximating the surface integrals by a numerical quadrature $\int_{S} f(\mathbf{x}) \mathrm{dS} \approx \sum_{m=1}^{M} \alpha_{m} f\left(\mathbf{x}_{m}\right)$ and using the Dirac delta function property $f(x)=\int_{V} f\left(x^{\prime}\right) \delta\left(x^{\prime}-x\right) \mathrm{dV}$ we get

$$
\begin{aligned}
& u_{i}^{b}(\mathbf{x}, t) \approx \\
- & \sum_{m=1}^{M} \int_{-\infty}^{\infty} d \tau \int_{V^{s}} \alpha_{m} n_{j}^{\prime} C_{i j k l} \partial_{k} u_{l}^{b}\left(\boldsymbol{\xi}_{m}, \tau\right) G_{i n}\left(\boldsymbol{\xi}_{m}, t-\tau ; \mathbf{x}, 0\right) \delta\left(\xi-\mathbf{x}_{m}\right) \mathrm{d}\left(V^{s}\right) \\
+ & \sum_{m=1}^{M} \int_{-\infty}^{\infty} d \tau \int_{V^{s}} \alpha_{m} n_{j}^{\prime} C_{i j k l} u_{i}^{b}\left(\boldsymbol{\xi}_{m}, \tau\right) G_{k n}\left(\boldsymbol{\xi}_{m}, t-\tau ; \mathbf{x}, 0\right) \partial_{l} \delta\left(\boldsymbol{\xi}-\mathbf{x}_{m}\right) \mathrm{d}\left(V^{s}\right)
\end{aligned}
$$

for $\mathbf{x}_{m}$ on $\partial V^{s}$.

Expression (6) has the form of a body-force contribution with a discrete distribution of moment tensor and force sources on $\partial V^{s}$, and it completely represents the background wavefield in an unmodified model in subdomain $V^{s}$. It is similar to the so called "time reversal mirrors" in acoustics [23, 41], although with reconstructing the wavefield forward in time rather than refocussing it backward in time. Modifying the structure in $V^{s}$ and imposing the same moment tensor and force sources on the boundary results in wavefield reconstruction with additional scattering terms superimposed on the background field: $\mathbf{u}(\mathbf{x}, t)=$ 
$\mathbf{u}^{b}(\mathbf{x}, t)+\mathbf{u}^{s}(\mathbf{x}, t)$. As before, absorbing boundary conditions are necessary on the representation of $\partial V^{s}$ in the local solver to absorb $\mathbf{u}^{s}(\mathbf{x}, t)$ and avoid non-physical reflections off model boundaries.

The equivalence between the distribution of body forces and introducing a discontinuity on the surface $\partial V^{s}$ in equation (3) is discussed in Chapter 3 of Aki and Richards [1] in the context of representing finite faults.

\subsection{Extrapolation}

Wavefield extrapolation propagates the wavefield from a local domain through an encompassing background domain. The interactions in the vicinity of the source are accounted for in the local solver and carried over to receivers at distance. Like wavefield injection, extrapolation consists of two steps. First, the source region is simulated in the 3-D solver and the fields are recorded on the boundary. Next, that local boundary is fictitiously represented in the background medium where it introduces the local wavefields.

As before, let $V$ be a linearly elastic background model subdivided by a fictitious interface $\partial V^{s}$ such that $V^{s}$ is the region of the local truncated volume embedded in $V$, and $V=V^{b} \cup V^{s}$ (Fig. $2 b)$. This time, however, the representation of $V^{s}$ in the local solver includes the seismic source. In the first step, the local wavefield is recorded on $\partial V^{s}$. In the second step, the boundary $\partial V^{s}$ is fictitiously represented in the background medium and allows for extrapolation to receivers. If the model in the local domain remains unchanged relative to the representation of $V^{s}$ in the background model, the coupling should reproduce the original background wavefields in $V^{s}$, as $\mathbf{u}^{s}(\mathbf{x}, t)=0$ and $\mathbf{u}(\mathbf{x}, t)=\mathbf{u}^{b}(\mathbf{x}, t)$. With the structure in the local representation of $V^{s}$ modified to include heterogeneities, the total wavefield $\mathbf{u}(\mathbf{x}, t)$ in the local solver and at receivers after extrapolation becomes $\mathbf{u}(\mathbf{x}, t)=\mathbf{u}^{b}(\mathbf{x}, t)+\mathbf{u}^{s}(\mathbf{x}, t)$, where $\mathbf{u}^{s}(\mathbf{x}, t)$ is the locally scattered wavefield.

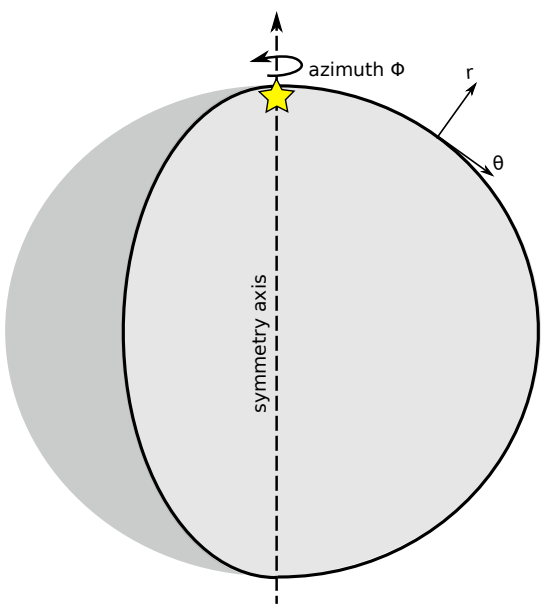

Figure 3: Sketch of an Earth model with axisymmetric geometry used in AxiSEM. The sphere is collapsed to a 2-D disk, while the azimuthal dimension is tackled analytically to generate 3 $\mathrm{D}$ wavefields in a 1-D Earth. The 3-D equations of motion are re-cast into a suite of 2-D problems with the source on the symmetry axis. [47]
We can repropagate the scattered wavefield out of the local domain $V^{s}$ to the receivers in the background domain $V^{b}$ by discretising surface integrals in the representation theorem like in Section 2.1.2, and imposing moment tensor and force sources on the fictitious representation of $\partial V^{s}$ in the background model. The total wavefield at the receivers at distance is the sum of the background and scattered wavefields, $\mathbf{u}(\mathbf{x}, t)=\mathbf{u}^{b}(\mathbf{x}, t)+\mathbf{u}^{s}(\mathbf{x}, t)$. We know the background wavefield $\mathbf{u}^{b}(\mathbf{x}, t)$ in $V^{b}$ and on $\partial V^{s}$, and hence we wish to represent the scattered wavefield $\mathbf{u}^{s}(\mathbf{x}, t)$ in $V^{b}$ in order to define the total displacement $\mathbf{u}(\mathbf{x}, t)$ at the receivers in $V^{b} . \mathbf{u}^{s}(\mathbf{x}, t)$ does not have sources in $V^{b}$, so $\mathbf{f}^{s}(\mathbf{x}, t)=$ $0 \forall \mathbf{x} \in V^{b}$ and the volume integral vanishes. As mentioned above, integrals over the surface reduce to integrals over $\partial V^{s}$. Repeating the procedure from Section 2.1.2 for the scattered field in $V^{b}$, with the numerical quadrature and the delta property of the Dirac delta we get

$$
\begin{aligned}
& u_{n}^{s}(\mathbf{x}, t) \approx \\
& \sum_{m=1}^{M} \int_{-\infty}^{\infty} d \tau \int_{V^{b}} \alpha_{m} n_{j} C_{i j k l} \partial_{k} u_{l}^{s}\left(\boldsymbol{\xi}_{m}, \tau\right) G_{i n}\left(\boldsymbol{\xi}_{m}, t-\tau ; \mathbf{x}, 0\right) \delta\left(\boldsymbol{\xi}-\mathbf{x}_{m}\right) \mathrm{d}\left(V^{b}\right) \\
& -\sum_{m=1}^{M} \int_{-\infty}^{\infty} d \tau \int_{V^{b}} \alpha_{m} n_{j} C_{i j k l} u_{i}^{s}\left(\boldsymbol{\xi}_{m}, \tau\right) G_{k n}\left(\boldsymbol{\xi}_{m}, t-\tau ; \mathbf{x}, 0\right) \partial_{l} \delta\left(\boldsymbol{\xi}-\mathbf{x}_{m}\right) \mathrm{d}\left(V^{b}\right)
\end{aligned}
$$

for $\mathbf{x}_{m}$ on $\partial V^{s}$. Note that for a domain at the surface around the source region, the total wavefield from the local solver can be considered as the locally scattered wavefield $\mathbf{u}^{s}(\mathbf{x}, t)$, since all path effects are accounted for in the extrapolation step. For the more general case of a local domain along the propagation path, however, the locally scattered wavefield needs to be separated from the background medium in order to account for the propagation effects of the background wavefield outside of the local region.

Expression (7) has the form of a body-force contribution with a discrete distribution of moment tensor and force sources on $\partial V^{s}$. In other words, we can regenerate the scattered field $\mathbf{u}^{s}$ at any point $\mathbf{x} \in V^{b}$ if we know the scattered field on $\partial V^{s}$ and the Green's functions between $\mathbf{x}$ and $\partial V^{s}$.

\section{IMPLEMENTATION}

The proposed implementation of hybrid coupling embeds any local 3-D domain in a 1-D spherically symmetric Earth model. We treat the global background medium using AxiSEM [48] and Instaseis [63] both to extract the background wavefields necessary for injection in the local domain and to extrapolate the scattered wavefields back to receivers on the Earth's surface. The approach is centred around precomputed databases that provide the general framework of the method and leave flexibility for coupling with an arbitrary local solver of choice.

\subsection{AxiSEM: 2.5-D global wavefields}

AxiSEM is a parallel spectral element axisymmetric solver based on the variational form of the wave equation. Simulations are run in two-dimensional (2-D) domains, while the third azimuthal dimension is tackled analytically to generate 3 -D wavefields in 1-D Earth models, as shown in Fig. 3. Such an axisymmetric setting reduces the computational cost by up to 3-4 orders of magnitude relative to full 3-D modelling [48]. With AxiSEM 
simulations scaling with frequency to the third power, it allows for global wave propagation at frequencies above $1 \mathrm{~Hz}$ with a reasonable computational effort. Should only source-receiver pairs with distances under 180 degrees be of interest, the numerical cost can be further limited by truncating the 2-D slice to a given distance (which adds an absorbing boundary to the system). For example, limiting the domain to 90 degrees halves the computational requirements relative to a full Earth simulation.

Apart from a compromise on model symmetry, the solver does not make any limiting assumptions regarding short-period wave propagation physics (the lack of rotation and gravity is not relevant for periods below $100 \mathrm{~s}$ ) or on the source radiation pattern. Transverse anisotropy - the most complex type of anisotropy for a spherically symmetric model [61] - and attenuation [62] can also be accounted for.

\subsection{Instaseis: a database approach}

The AxiSEM-generated Instaseis databases of 2-D Green's functions act as a once-and-for-all solution to wave propagation in spherically symmetric models and store the basis coefficients of Lagrange polynomials, typically of degree 4 . The stored databases give flexibility with respect to parameter alterations, such as modifications in source properties (radiation patterns, source-time function), in the source-receiver geometry, and in local domain dimensions and location (including regions around the source or receivers, and at depth). Green's functions for a given Earth model therefore allow for a wide range of parameter changes in subsequent hybrid applications without the need of re-running the global simulation. We distinguish two types of Instaseis databases:

1. The forward database is generated by a moment tensor point source at a fixed depth. For such Green's functions receivers exist throughout the medium, whereas the source can be rotated from the north pole to any longitude and latitude. However, it remains at the depth prescribed at the time of the simulation.

2. The reciprocal database is generated by a single force point source at a fixed depth and recorded throughout the medium. Due to the reciprocity of Green's functions, the locations of the single force source can be treated as three component receivers. Therefore, in such databases the sources exist throughout the medium, whereas receivers can be rotated to any longitude and latitude, but must remain at the depth prescribed at the time of the simulation. Typically, reciprocal databases are generated by a single force on the Earth's surface which mimics real-world receivers.

Instaseis - the backbone of our method - is a tool that efficiently accesses, processes, and extracts full 3-D wavefields from such pre-computed AxiSEM databases. Given the $4^{\text {th }}$ order spatial accuracy of the Green's functions, Instaseis can interpolate the wavefields to a mesh-independent location, while higher-order Lanczos resampling allows to retrieve seismograms at any sampling rate. Changing the source radiation pattern or the sourcetime function, or defining a finite fault does not require a new database generation, as Instaseis convolves chosen source parameters into the Green's functions. In the following we discuss how the two classes of Instaseis databases serve as a basis for the global hybrid framework.

\subsection{Hybrid: a flexible framework}

The Instaseis-based approach to global hybrid modelling exploits the reciprocity of Green's functions to accommodate both injection and extrapolation of wavefields. Each of the database types imposes different geometrical source-receiver constraints, but nonetheless both represent the same response of the same medium (for a given spherically symmetric model and a given frequency). A forward database allows to place receivers anywhere in the domain and requires a fixed source depth, and therefore lends itself to wavefield injection that necessitates the response of the background medium on the boundary of the local domain. A reciprocal database, on the other hand, allows to place sources anywhere in the domain and fixes receivers at the surface, and is appropriate for wavefield extrapolation.

As discussed, three coupling scenarios are possible (Fig. 1). In the most general case of a complex subdomain at depth, we identify the proposed global hybrid method as a three-step approach (with the other cases reducing to two steps, steps 2 \& 3 and steps $1 \& 2$ for local domains around the source and receiver, respectively):

1: [Instaseis injection] The forward database is used to extract the background wavefield on an artificial boundary which is defined by the mesh of the local method chosen for the coupling. The incoming wavefield is then imposed on those boundary points of the 3-D solver and drives the local wave propagation.

2: [Local solver] The total wavefield from the simulation is recorded on the bounding box. The locally scattered wavefield is equal to the difference between the total local wavefield and the background wavefield $\left(\mathbf{u}^{s}(\mathbf{x}, t)=\mathbf{u}(\mathbf{x}, t)-\mathbf{u}^{b}\right)$.

3: [Instaseis extrapolation] The locally scattered field is convolved with the reciprocal database of Green's functions and repropagated back to the receivers on the surface. It is then superimposed on the pre-computed background wavefield to render the total global wavefield that incorporates global propagation effects as well as local 3-D scattering.

The availability of both forward and reciprocal databases of Green's functions for a global model renders the method particularly attractive. The spherically symmetric set-up caters for a broad range of possible geometries and sources with only two database simulations - the same databases can be queried for any arbitrary source-receiver configurations. The heavy computational burden of simulating the background field is therefore transposed to storage requirements and to the embarrassingly parallel and thus massively scalable procedure of accessing a relevant database to extract and process wavefields.

The modified hybrid Instaseis interface provides the processing infrastructure, with rotations between local and global coordinate systems, interpolations of wavefields to points defined by the mesh or quadrature of the local method used for the coupling, temporal resampling of the local and global wavefields, as well 
as changes in the source mechanism. Communication between Instaseis and the local solver happens via HDF5 files.

\subsection{Local solvers: SPECFEM3D Cartesian and Wave Propagation Program}

Currently the framework has been coupled with two local 3-D codes representative of the typical and most widely used methods using spectral elements and finite differences: Specfem3D Cartesian [32], a spectral-element (SEM) solver, and Wave Propagation Program (WPP) [46, 51], a second order finite-difference (FD) solver. The Specfem3D implementation includes all three coupling cases, with the choice of the local domain on the source side, on the receiver side, and at depth. The WPP implementation, a strictly cartesian code, has been developed for a specific source-side application [52, 53, in preparation] and has not been extended to the receiver and depth cases.

\subsubsection{Specfem3D Cartesian}

Specfem3D Cartesian is a software that is mostly used for seismic wave propagation at local and regional scales, although it can be applied to other acoustic and elastic wave propagation problems. It is well-suited for handling various geometries and allows to account for 3-D complexities affecting the seismic wavefield, such as lateral variations of elastic parameters and density, anelasticity, full 21-parameter anisotropy, and topography of internal discontinuities and of the free surface. It also includes coupled fluid-solid domains.

We inject the global wavefield into a Specfem3D simulation via imposing the Dirichlet boundary condition (see Section 2.1.1) on all points of the boundary of the SEM mesh. In order to avoid spurious reflections, we then apply the paraxial boundary condition [57] to absorb the residual outgoing scattered wavefield. Applying the boundary condition to the outgoing scattered wavefield requires the knowledge of background tractions on the boundary of the SEM mesh - the known background tractions are subtracted from the locally computed tractions, while the residual (i.e. the difference not known a priori) is absorbed. Therefore, we query a forward Instaseis database for velocities and tractions on the GLL points on the mesh boundary. The values are stored in HDF5 files and later accessed by Specfem3D during the simulation - a modification added to the code for coupling purposes. Note that absorbing only the lower energy residual scattered wavefield renders the boundary condition more effective.

For extrapolation we save displacements and stresses from the local simulation into interfacing HDF5 files. Given the mesh construction, there is no need to define a new quadrature to discretise the integral in the representation theorem and we directly use the weights of the GLL points in the extrapolation equation 7 . The integration points are therefore well-defined for a high order quadrature which renders the extrapolation step of the coupling efficient and easy to implement. Moreover, the native mesher of the code respects the curvature of the Earth's surface as well as of the 1-D interfaces.

\subsubsection{Wave Propagation Program}

The Wave Propagation Program (WPP) is a second order (in time and space) open source FD solver developed at Lawrence
Livermore National Laboratory for local and regional seismic wave propagation. WPP solves the elastodynamic equations of motion for elastic or anelastic materials with fully 3-D heterogeneous material model specification, including anisotropy and anelastic attenuation with P- and S- wave quality factors. Surface topography can be represented through a boundary conforming curvilinear mesh created automatically with a built-in mesh generator from user-supplied files. Supergrid absorbing boundary conditions are implemented to dampen the outgoing wavefields, and depth-dependent mesh refinement can account for the increase in seismic wavespeeds.

For the extrapolation step necessary for the source-side coupling, we save displacements and strains from the local simulation into interfacing HDF5 files. Given the Cartesian mesh construction, we need to define a quadrature to discretise the integral in the representation theorem. With a dense regular FD grid, we choose to use the mid-point rule with the areas between grid points as weights in equation 7 . In the current implementation mesh construction does not respect the curvature of the Earth's surface or of the 1-D interfaces.

\section{VAlidation}

To benchmark the hybrid scheme we first consider the Specfem3D Cartesian implementation and reconstruct the background wavefield for a 1-D model from the original Instaseis database. We then introduce a smooth Gaussian perturbation in the local Specfem region and benchmark hybrid seismograms against a 3-D reference solution computed by Specfem3D Globe. Finally, we turn to the WPP-Instaseis source-side implementation at high frequencies and reconstruct 1-D wavefields. All 1-D simulations that reconstruct the background wavefield were validated for multiple receiver locations and components. The 3-D validation, however, was constrained to receiver locations at 30 degrees due to computational costs of global Specfem3D Globe simulations. We chose 30 degrees as a compromise between the simulation time of Specfem3D Globe and our aim to validate waveforms at teleseismic distances.

The accuracy of the benchmarks, and more generally of the hybrid method, depends on the meshing of the local solver relative to the 'dominant period' of the database. The parameters of the local simulation need to be considered carefully both relative to the requirements of the local solver itself, as well as relative to the discretisation of the surface integrals of the representation theorem for extrapolation and for injection via a distribution of point sources. Each use case needs to be considered separately, and it is recommended to test every problem-specific coupling set-up with 1-D benchmarks before introducing 3-D structures.

The dominant frequency (inverse of the dominant period) in this paper is understood as the frequency corresponding to the halfmaximum of the spectral amplitude of the source-time function, ensuring that the bulk of the energy up to the dominant frequency is contained in the simulations. In a Specfem3D Globe nomenclature, on the other hand, frequency corresponding to the maximal frequency with non-zero spectral amplitude is considered as the 'dominant frequency'. The $10 \mathrm{~s}$ dominant period runs discussed below, for example, would be referred to as ' $5 \mathrm{~s}$ ' in Specfem3D Globe. 


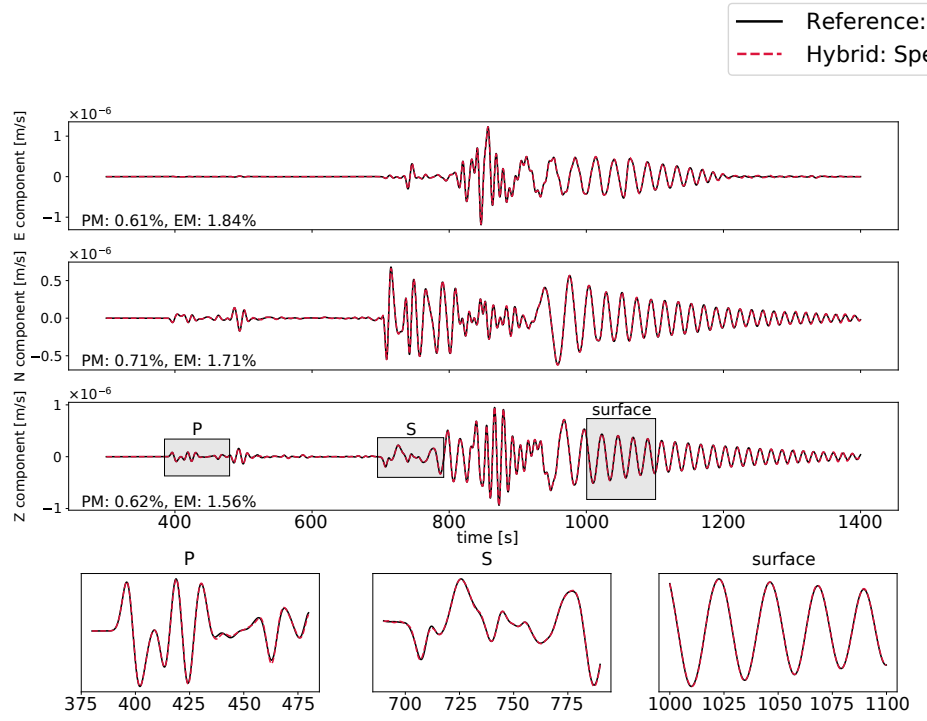

(a)

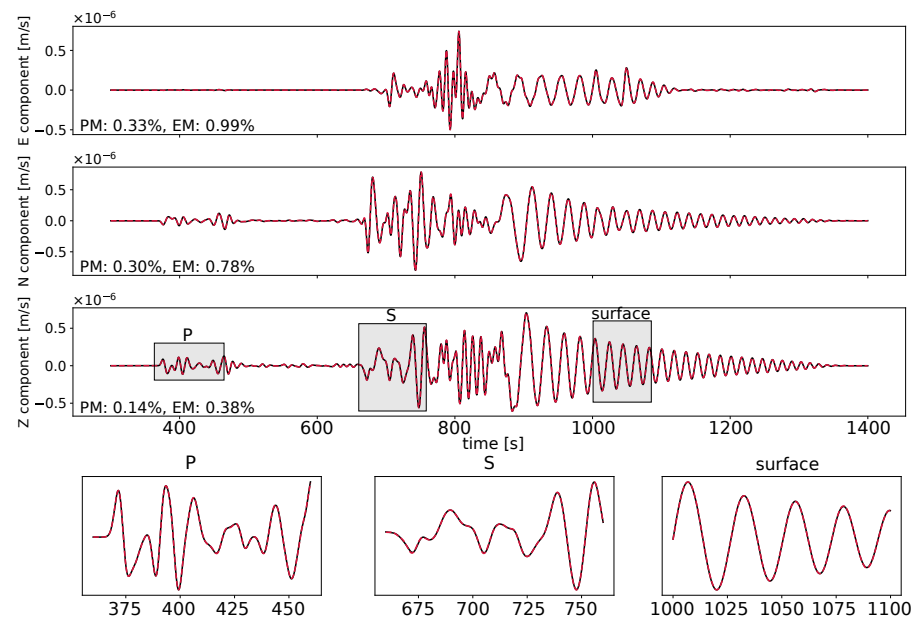

(b)

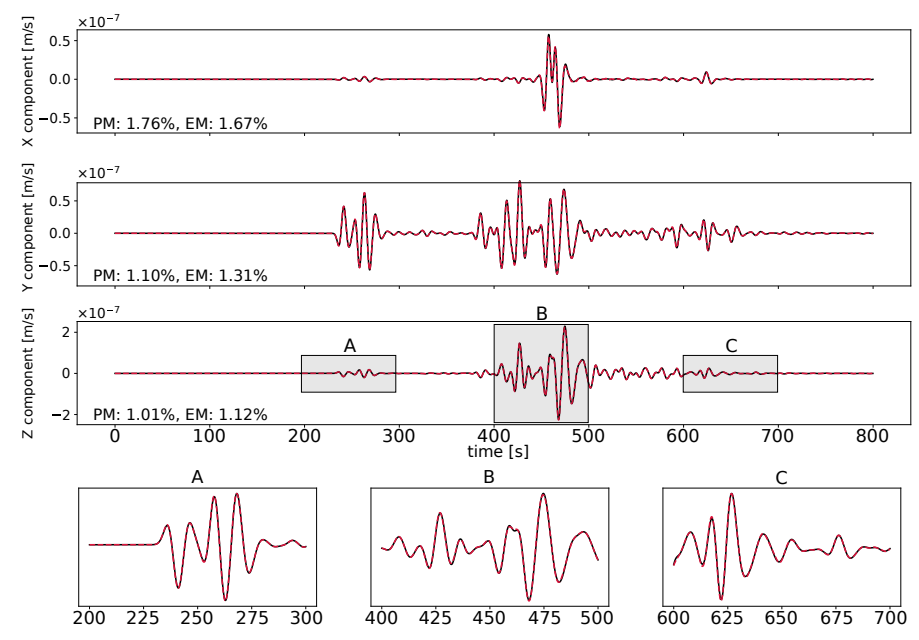

(c)

Figure 4: Examples of velocity benchmark waveforms for a 1-D PREM model with a local Specfem3D Cartesian domain (a) at the surface around the source, (b) at the surface under the receivers, and (c) at depth along the propagation path. All benchmarks include three component seismograms of the original 1-D waveform from the Instaseis database (black line) and a Specfem-Instaseis hybrid reconstruction (dashed red line). Benchmark in (c) reconstructs wavefields on the boundary of the local domain at depth and validates only the injection step for a buried domain. Extrapolation to receivers at distance cannot be tested for this set-up with a 1-D model (see main text). Presented waveforms are for a full moment tensor source with a Gaussian source-time function of a 5-second half-width (10 s dominant period of seismograms), and PREM model does not include attenuation. 


\subsection{1-D benchmarks: Specfem-Instaseis wavefield reconstruction}

The hybrid methodology should reproduce the original background wavefields if the local domain remains unmodified relative to the background model. With the local domain at the surface, either around the source or under the receivers, the wavefields can be reconstructed at teleseismic distances. However, for the local domain at depth along the propagation path, only the "residual" scattered wavefield $\left(\mathbf{u}^{s}(\mathbf{x}, t)\right)$ is extrapolated to receivers at distance, while unchanged 1-D models only regenerate the 1-D interactions. Hence, the 1-D benchmark for the local domain at depth can only prove a correct reconstruction of the 1-D wavefield inside of a buried domain without a free surface. It tests a correct implementation of wavefield injection at depth, as extrapolation for the depth case cannot be validated with a 1-D model.

The accuracy is quantified in terms of phase and envelope misfits as defined by Kristeková et al. [34] (PM and EM, respectively), where misfits below $20 \%$ represent an 'excellent' fit, while misfits in the 20-40\% range represent a 'good' fit. For each pair of synthetics (hybrid Specfem-Instaseis versus 1-D), we compute the globally normalized EM and PM. Fig. 4 presents sample velocity benchmark waveforms for a 1-D PREM model with a Gaussian source-time function of a 5 second half-width (i.e. 10 s dominant period) and a full moment tensor source. The local Specfem domains are 16 wavelengths in $x, y$ and $z$-dimensions chosen to encompass a reasonable Gaussian perturbation of the 1-D model in subsequent 3-D benchmarks - and the mesh is constructed with 1.5 elements per wavelength. For $4^{\text {th }}$ order spectral elements, this results in about 7 points per wavelength (PPW) The 1-D PREM model does not include attenuation for later ease of 3-D benchmarking against Specfem3D Globe, as different implementations of attenuating properties of the medium can add to waveform discrepancies. The 1-D PM and EM turn out invariably small $(<2 \%)$ with the $1-\mathrm{D}$ hybrid waveforms indistinguishable from the original background waveforms.

\subsection{3-D benchmarks: Specfem-Instaseis local scattering}

To benchmark the hybrid framework for 3-D structures in the local domain, we introduce a $40 \%$ Gaussian perturbation of seismic velocities $v_{p}, v_{s}$, and of density $\rho$ over PREM into the Specfem3D Cartesian domains at depth and on the surface, around the source and under the receivers. The extreme perturbation, rarely observed in bulk-Earth applications, has been chosen to validate the accuracy of our method as an upper bound. As before, a full moment tensor source and a Gaussian sourcetime function of a 5 second half-width are used, resulting in wavefields with a dominant period of $10 \mathrm{~s}$. The Gaussian perturbation is defined with a standard deviation equal to two $\mathrm{S}$ wave wavelengths. For the domain at depth, the perturbation is located in the middle of the domain in $x, y$ and $z$, and the domain itself is centred around the turning point of the $\mathrm{P}$ and $\mathrm{S}$ wave for the presented source-receiver configuration of 30 degrees epicentral distance (Fig. 5a). For the domains at the free surface - around the source and under the receivers - the centre of the Gaussian is located in the middle of the domain in $x$ and $y$ ), and at a depth equal to two standard deviations, resulting in a strong perturbation on the surface (Fig. 5b). The domains are $16 \times 16 \times 16$ wavelengths in size, which is equivalent to $8 \times 8 \times 8$ standard deviations of the Gaussian perturbation. This ensures that the centre of the perturbation is 4 standard deviations from each boundary, leaving the boundary unaltered relative to the 1-D PREM model. A for the 1-D benchmarks, the mesh is constructed with 1.5 elements per $\mathrm{S}$ wavelength (i.e. 7 PPW).

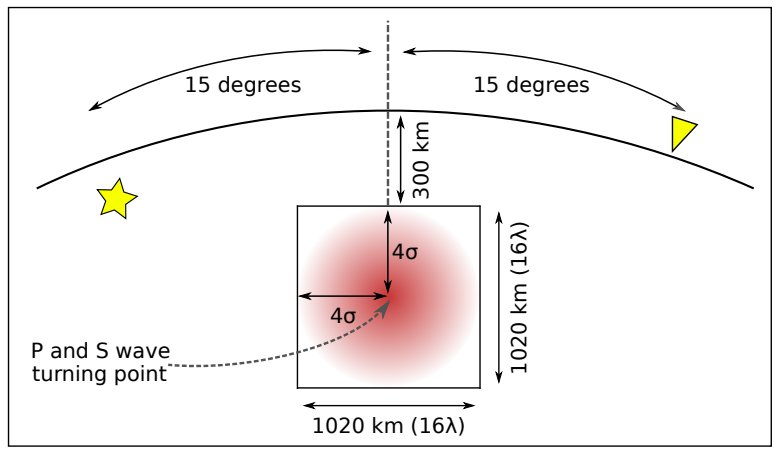

(a)

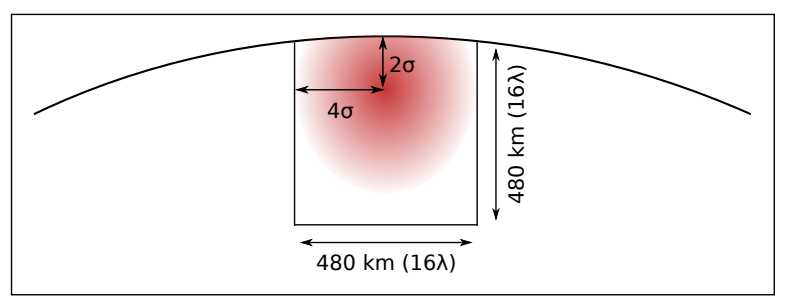

(b)

Figure 5: Sketch of benchmark set-ups of the Specfem-Instaseis hybrid coupling with a $40 \%$ Gaussian perturbation of seismic velocities $v_{p}, v_{s}$, and of density $\rho$. The domains are $16 \times 16 \times 16$ wavelengths in size, which is equivalent to $8 \times 8 \times 8$ standard deviations of the Gaussian perturbation. This ensures that the centre of the perturbation is 4 standard deviations from each boundary, so that the boundary matches the 1-D background model. (a) The domain along the propagation path is centred around the turning point of the direct $\mathrm{P}$ and $\mathrm{S}$ waves for the source-receiver configuration of 30 degrees epicentral distance. The top of the domain is $300 \mathrm{~km}$ under the Earth's surface. The standard deviation of the Gaussian perturbation is equal to approximately two $\mathrm{S}$ wavelengths in the domain, i.e. 130 $\mathrm{km}$. (b) For domains at the free surface - around the source and under the receivers - the centre of the Gaussian is located in the middle of the domain in $x$ and $y$, and at a depth equal to two standard deviations, resulting in a strong perturbation on the surface. The standard deviation of the Gaussian perturbation is equal to approximately two $\mathrm{S}$ wavelengths in the domain, i.e. $60 \mathrm{~km}$.

Fig. 6 presents sample velocity benchmark waveforms for the 1-D PREM model with a $40 \%$ Gaussian perturbation (hybrid Specfem-Instaseis versus Specfem3D Globe). While the 1-D benchmark for a buried domain along the propagation path is not complete, as extrapolation to receivers cannot be tested by construction, for the 3-D case it presents the most complete setting that validates both injection (i.e. coupling with a forward database) and extrapolation (i.e. coupling with a reciprocal database). The global wavefield for the depth perturbation has 


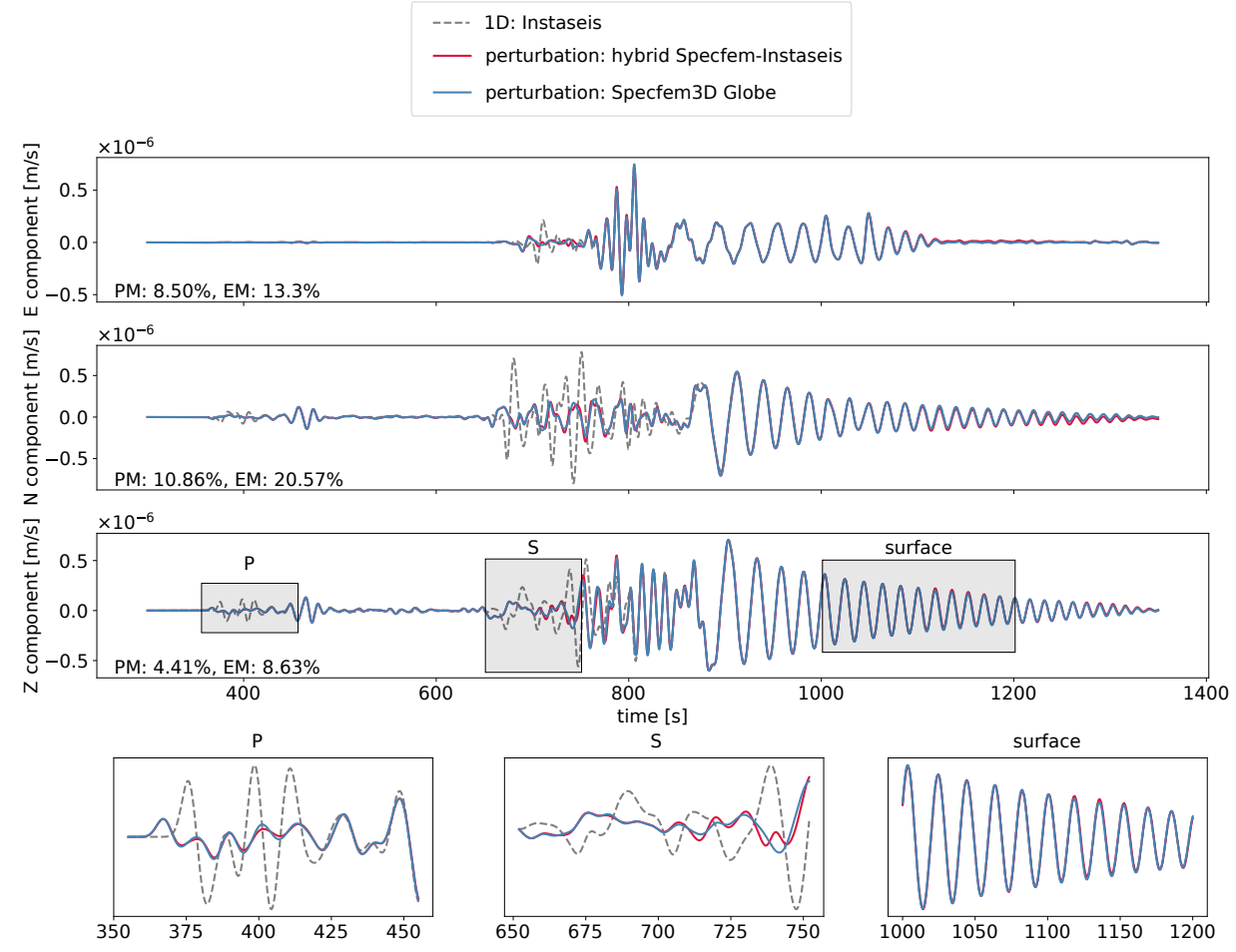

(a) run ID: 1
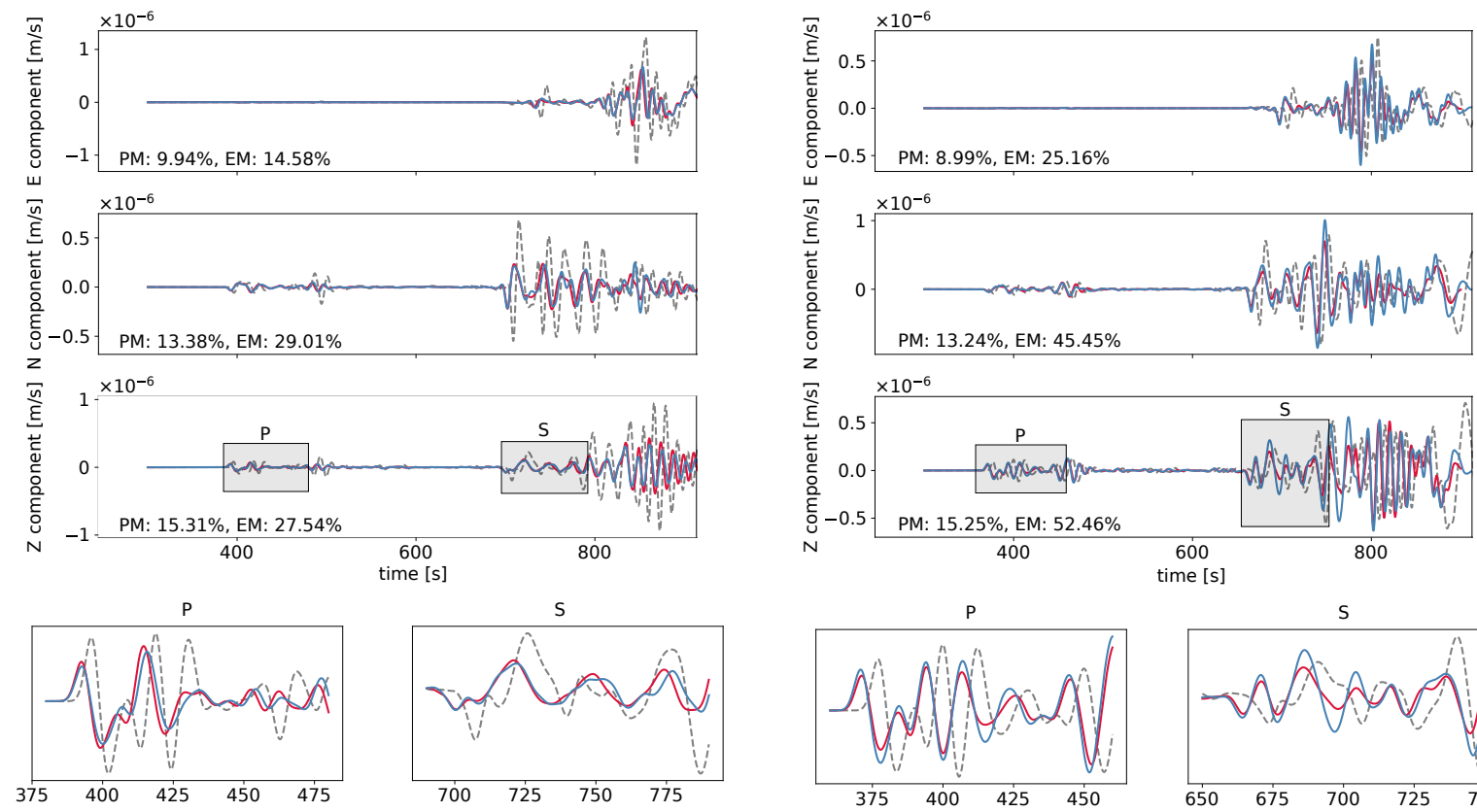

(b) run ID: 2

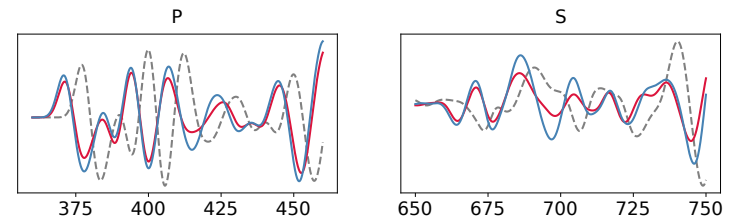

(c) run ID: 3

Figure 6: Examples of velocity benchmark waveforms of a local Specfem3D Cartesian coupling for a 1-D PREM model with a $40 \%$ Gaussian perturbation (a) at depth along the propagation path, (b) at the surface around the source, and (c) at the surface under the receivers. All benchmarks include three component seismograms of the original 1-D waveform from the Instaseis database (dashed grey line), a Specfem-Instaseis hybrid perturbation (red line), and a Specfem3D Globe perturbation (blue line). Benchmark in (a) validates both injection and extrapolation and presents a full seismogram including surface waves, while (b) and (c) are limited to body waves (see main text for an explanation). Presented waveforms are for a full moment tensor source with a Gaussian source-time function of a 5-second half-width (10 s dominant period of seismograms), and PREM model does not include attenuation. Run ID in each subfigure caption corresponds to IDs in Table 2 with a summary of computational costs. 
therefore been simulated for 23 minutes to obtain a complete seismogram at 30 degrees, and Fig. 6a presents the resulting benchmark of the full synthetic signal. Given the high computational cost of Specfem3D Globe simulations (271,200 CPUh for 23 minute seismograms at $10 \mathrm{~s}$ dominant period) and the completeness of the depth benchmark for injection and extrapolation, we choose to validate the source and receiver cases without surface waves and compute only 15 minutes of Specfem3D Globe seismograms. Moreover, since a correct generation of realistic surface waves requires a full 3-D representation of the crust between the source and the receiver, our hybrid methodology that approximates the Earth as a spherically symmetric layered medium with a flat free surface is not well suited for surface-wave applications. Since the hybrid benchmark at depth is complete and fully sufficient, and given our focus on highfrequency body waves, a validation of 1-D surface waves for all three coupling cases is of little interest.

The model perturbation has a comparable effect in both solvers, with differences arising due to numerical reasons as well as limitations of the hybrid method. The numerical issues, such as distinct meshing schemes, source and receiver mislocations, and accumulative round-off errors, are a consequence of solver differences. According to Leng et al. [35], the misfits between Instaseis databases (that correspond to AxiSEM3D solution to 1-D models) and Specfem3D Globe simulations can generally be expected to remain below $2 \%$ for 1-D models, although envelope misfits can sometimes be very large for unidentified reasons For 3-D tomographic models, the misfits between AxiSEM3D and Specfem3D Globe are more significant and vary between receiver locations. Thus, with a locally perturbed 1-D model we can expect a mismatch between the hybrid and full 3-D seismograms, as meshing differences alone can result in different representations of the perturbation. Moreover, in the local method of the hybrid scheme, it is necessary to use absorbing boundary conditions which are known to be imperfect and can result in often significant spurious reflections. On top of such numerical issues, the limitations of a one-way hybrid scheme are expected to contribute to the visible waveform discrepancies. Higher order long range scattering is not accounted for in the coupling, while the Specfem3D Globe waveform represents all interactions between the perturbation, the free surface, and the internal 1-D model discontinuities.

The benchmark for the perturbation at depth along the propagation path shows the best fit, with PM of $10 \%$ and less and EM of $20 \%$ or less - values considered to represent an 'excellent fit' by Kristeková et al. [34]. With the local domain of 16 wavelengths in size (1020 km in $x, y$ and $z)$ and centred around the turning point of $\mathrm{P}$ and $\mathrm{S}$ waves, most contributing long range scattering is accounted for due to the size of the domain, and the resulting misfits can be mostly attributed to numerical discrepancies discussed above. For the source and receiver cases, however, the local domain is significantly smaller $(480 \mathrm{~km}$ in $x, y$ and $z$ ), as the decrease in wave velocities results in shorter wavelengths close to the Earth's surface. The local domain, therefore, does not encompass all contributing long range scattering and results in larger differences and breaks the one-way assumption of the method. Filtering all components at 20 seconds reduces the misfits for all three cases, though the visible amplitude differences for the source and receiver coupling settings remain The extreme $40 \%$ perturbation - validating the accuracy of our method as an upper bound - can be benchmarked with a 'good fit' (as defined by Kristeková et al. [34]) for all three coupling set-ups, and we expect the higher order long range scattering from more realistic structures to remain small at teleseismic distances relative to primary interactions of interest. Taking all contributing factors into consideration, we deem the results acceptable with the PM and EM under $20 \%$ for the complete coupling case that incorporates a perturbation at depth, with the larger misfits for the coupling set-ups under the free surface attributed to the limitations of the method.

\subsection{High-frequency WPP-Instaseis wavefield reconstruction}

The current implementation of the WPP-Instaseis source-side coupling does not respect the curvature of the Earth's surface or of internal discontinuities. A regular Cartesian WPP mesh is used, which for large domains results in a significant difference in altitude between the spherical Earth and the flat Cartesian surface. The method, however, requires that the coupling surface $\partial V^{i}$ remains unchanged relative to the background model. For large WPP Cartesian domains, this condition is not met and numerical errors arise. We have found that the magnitude of resulting errors is also dependent on the size of the altitude gap relative to the wavelength.

For small domains on the order of tens of kilometres, the errors remain small. Given the cost of global Specfem 3-D simulations, however, our 3-D benchmarks are limited to a dominant period of 10 seconds. The local domain needs to be large enough to encompass a smooth Gaussian perturbation of two wavelengths, which for a 10-second simulation means $500 \mathrm{~km}$ in $x, y$, and $z$. In a domain of this size the model mismatch is large, as both the free surface and five discontinuities that are within the top $500 \mathrm{~km}$ of a 1-D model are misrepresented on a Cartesian mesh relative to a spherical background model.

We therefore choose to benchmark the WPP-Instaseis wavefield extrapolation for a short period 1-D reconstruction at 2 seconds with a local domain of size $30 \times 30 \times 30 \mathrm{~km}$. According to the WPP manual [51], the number of points per wavelength (PPW), the largest significant frequency of the source time function $\left(f_{\max }\right)$, the minimum wavelength $\left(v_{\min }\right)$ and the FD grid spacing $h_{\min }$ are related according to

$$
h_{\text {min }}=\frac{v_{\text {min }}}{\mathrm{PPW} * f_{\max }} .
$$

With a minimum wavespeed $v_{s}$ of $3360 \mathrm{~m} / \mathrm{s}, 16 \mathrm{PPW}$ recommended for best accuracy, and the required $f_{\max }$ of $1 \mathrm{~Hz}(1 \mathrm{~s}$, for a $2 \mathrm{~s}$ dominant period), the WPP simulation should be sampled with at least $210 \mathrm{~m}$ FD grid spacing in $x, y$ and $z$.

For the sake of variety, we choose an isotropic moment tensor source (explosion) and IASP91 as the 1-D background model, and we include attenuation to show the validity of the scheme for attenuating databases. As for the Specfem-Instaseis coupling in Section 4.1, the PM and EM are below 1\% (Fig. 7) for the chosen FD grid spacing of $200 \mathrm{~m}$. With such 1-D extrapolation benchmarks demonstrating successful coupling of WPP with the source-side framework, and with the Specfem-Instaseis 3-D benchmarks proving the validity of the method for 3-D structures in the local domain, a locally modified model can be considered accurate for WPP-Instaseis as long as the WPP implementation of 3-D model complexities is accurate. 

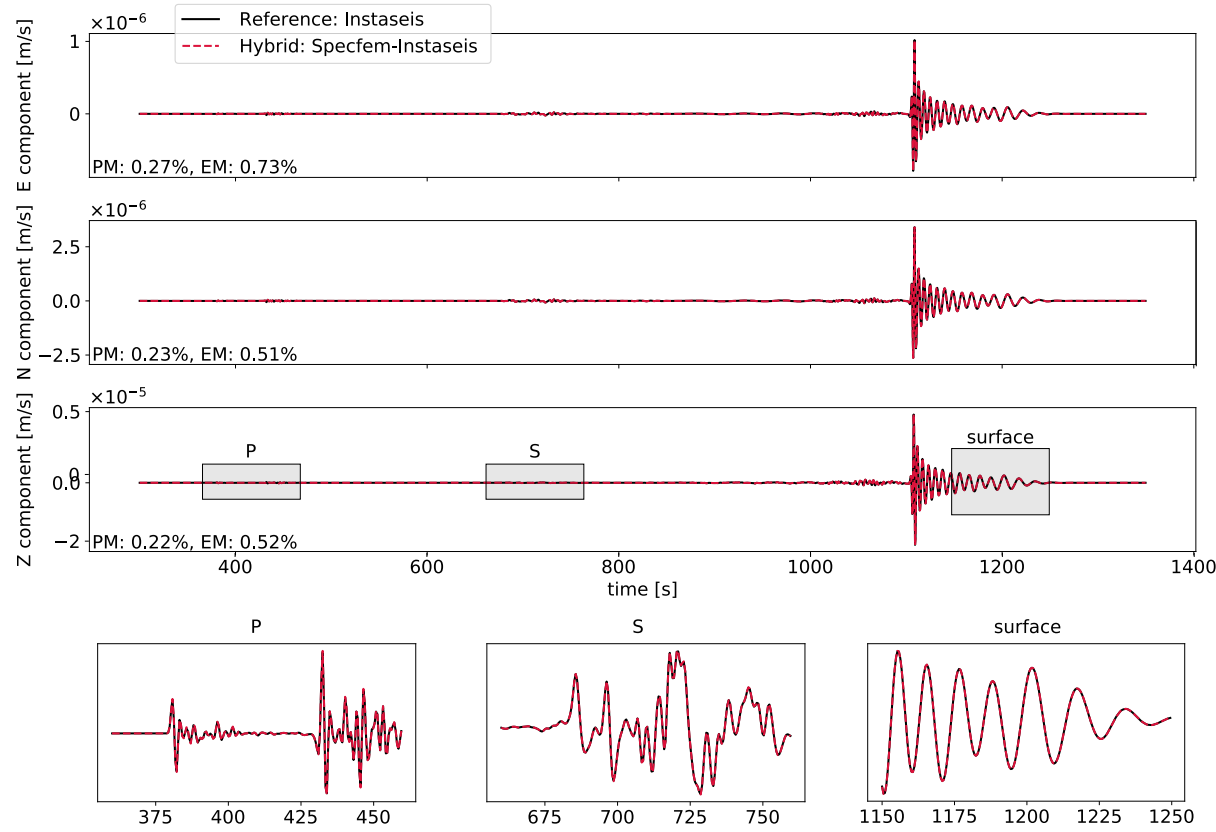

Figure 7: Example of velocity benchmark waveforms for a 1-D IASP91 model with a local WPP domain at the surface around the source (run ID: 4). The three component seismograms of a WPP-Instaseis hybrid reconstruction (dashed red line) are shown against the original 1-D waveform from the Instaseis database (black line). Presented waveforms are for an isotropic moment tensor source (explosion) with a Gaussian source-time function of a 1-second half-width ( $2 \mathrm{~s}$ dominant period of seismograms), and IASP91 model accounts for attenuation.

Table 1: Computational and storage requirements of Instaseis databases used for benchmarking

\begin{tabular}{cccccc}
\hline DB type & model & CPUh & Storage [GB] & Length [s] & Depths [km] \\
\hline BWD 10 s & PREM & 27 & 54 & 1500 & $0-1000$ \\
FWD 10 s & PREM & 57 & 114 & 1500 & $0-1000$ \\
BWD 2 s & IASP91 & n/a & 1380 & 3700 & $0-700$ \\
& (attenuating) & & & & \\
\hline
\end{tabular}

FWD stands for forward and BWD for reciprocal database, and database type also indicates the dominant period of the run. Maximum source depth, components, seismogram length and the range of epicentral distances define the final database size. All databases were generated for 3 components and all distances. Exact CPU hours of the 2 s reciprocal database are unknown, as it has been generated for other purposes prior to this study. 


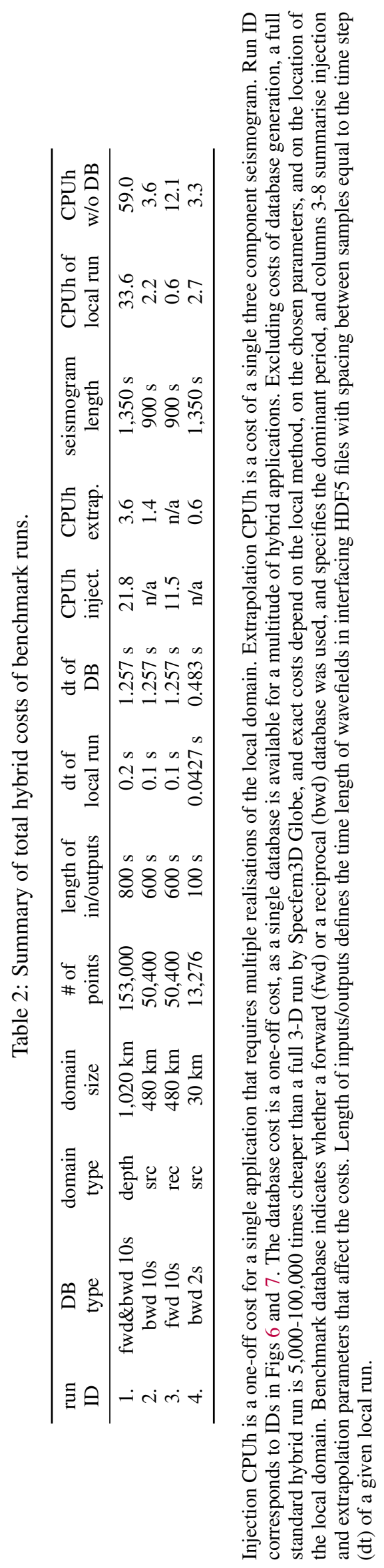




\subsection{Performance}

\subsubsection{Database generation and storage}

The bulk of the computational effort of the hybrid methodology lies in database generation, and the cost of generating an AxiSEM database scales with frequency to the $3^{\text {rd }}$ power [63]. The actual cost of each run depends both on the machine and on the chosen simulation parameters (e.g. attenuation, number of elements per wavelength), and a forward database is approximately twice as expensive as a reciprocal database for the same set of parameters. It should be noted that the cost can be significantly reduced for specialised studies with limited epicentral distances, as the 2-D disk in Fig. 3 can be cropped to encompass only the required latitudes (with absorbing boundaries applied to the additional non-physical boundary).

The advantage of database generation is the once-and-for-all approach. Storing the Green's functions from a single AxiSEM run, one can freely access and re-use the 1-D background wavefields for a variety of hybrid applications - the location and size of the local domain, and more generally of the source-receiver geometry can vary, and the source parameters can be modified Therefore, the cost of generating a database can be considered a one-off investment for a large spectrum of problems, shifting the focus from regular access to substantial computational resources to the necessary database storage requirements. As discussed by van Driel et al. [63] the storage space required for a reciprocal database depends on frequency content, maximum source depth, components, seismogram length and the range of epicentral distances. A forward database takes approximately twice as much space as a reciprocal database for the same set of parameters The CPU hours and storage requirements for the databases used in the benchmarks are listed in Table 1.

\subsubsection{Hybrid injection and extrapolation}

There is no general rule for the costs of injection and extrapolation, as both depend on the the length of the local simulation, as well as on mesh discretisation and time step of the local method:

- The number of points on the boundary of the local domain influences both injection and extrapolation. It depends on the size of the local domain, as well as on the points chosen for the coupling. For injection, the points must correspond to the mesh of the local method. For extrapolation, one could define a quadrature independent of the mesh points, provided that the local method can accurately interpolate the local wavefields to any point. In both Specfem and WPP implementations, however, the extrapolation points are chosen to be the local mesh points, and in WPP using only every other point on the boundary proves sufficiently accurate for extrapolation and reduces the cost.

- For injection, background wavefields extracted from the database are required to have the same time length and time step as defined in the local simulation, and both factors influence the cost. Longer time series require higher time to save to HDF5. Moreover, the costs of interpolating wavefields to the right time step vary and depend both on the sampling of the database and on the new sampling required.
- For extrapolation, the time length of the local wavefields influences the computational cost. However, the local wavefields are downsampled for extrapolation to avoid the high costs of interpolation, and hence the time step of the local simulation has less of an effect on the overall cost than in case of injection.

Table 2 summarises the injection and extrapolation costs and parameters (number of points, time steps, lengths of wavefields) for benchmark runs in Section 4.2, as well as CPU hours (CPUh) of respective local simulations and one-off database runs. Note that the cost of injection includes all receivers defined in the local domain, while the cost of extrapolation is a per receiver cost for a three-component seismogram. Therefore, the injection cost for a single application that requires multiple realisations of the same local domain is a one-off cost. Wavefields are saved into HDF5 files according to the requirements of the local mesh and remain available for multiple runs of the local solver, further reducing the costs of injection solely to the cumulative cost of required local runs. However, modifications in the local domain for hybrid extrapolation require re-running both the local simulation and the extrapolation to receivers.

Depending on the parameters of the set-up, the computational cost of a full standard hybrid run - including the cost of the local simulation that is method dependent, but excluding database generation - is reduced by a factor of 5,000-100,000 relative to a full 3-D run, provided that the local domain is of the order of tens of wavelengths in size. A Specfem3D Globe simulation for the 3-D benchmark at depth (run ID 1) cost 271,200 CPUh for 23 minutes of wave propagation, while the source and receiver cases (run ID 1 and 2, respectively) required 227,500 CPUh for 15 minutes of wave propagation. The corresponding hybrid coupling costs are on the order of tens of CPUh. The database runs, even though included in the comparison, are a one-off cost available for multiple hybrid applications and settings.

\section{WAVE-PROPAGATION APPLICATIONS}

Given its ability to generate high-frequency seismograms at teleseismic distances, the hybrid method allows to study the influence of localised 3-D structures on global propagation of body waves over $1 \mathrm{~Hz}$. Purely synthetic studies in multi-scale media are made possible, providing insights into the long-range effects of complex scattering and a better understanding of uncertainties in existing models. Realistic simulations of structure-induced anomalies, on the other hand, are essential to interpretation and analysis of seismic recordings, and can now be achieved for highest frequencies observed teleseismically.

\subsection{Source side heterogeneity}

With strong topography prevalent both in regions of high seismicity and in nuclear test sites, accounting for topographic scattering in a local 3-D model around the source region allows to capture its teleseismic effects on body waves - a topic further addressed by Pienkowska et al. [52, 53, in preparation] in the context of forensic seismology, where observations are compared with synthetics at $2 \mathrm{~Hz}$. Figure 8 illustrates the comparison on three locations in a USSR Degelen Mountain nuclear test site, demonstrating that topography alone can reproduce some 
SYNTHETICS
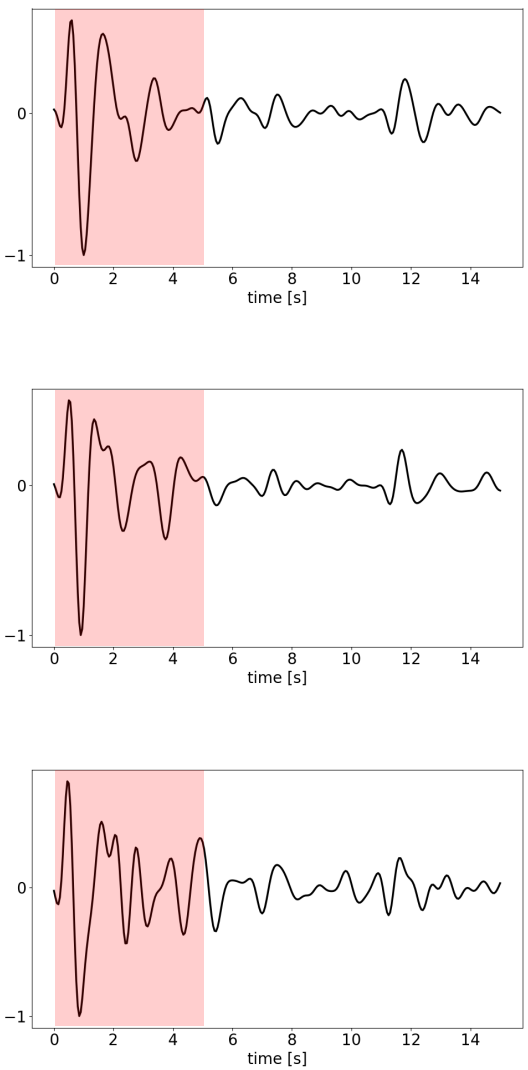

OBSERVATIONS
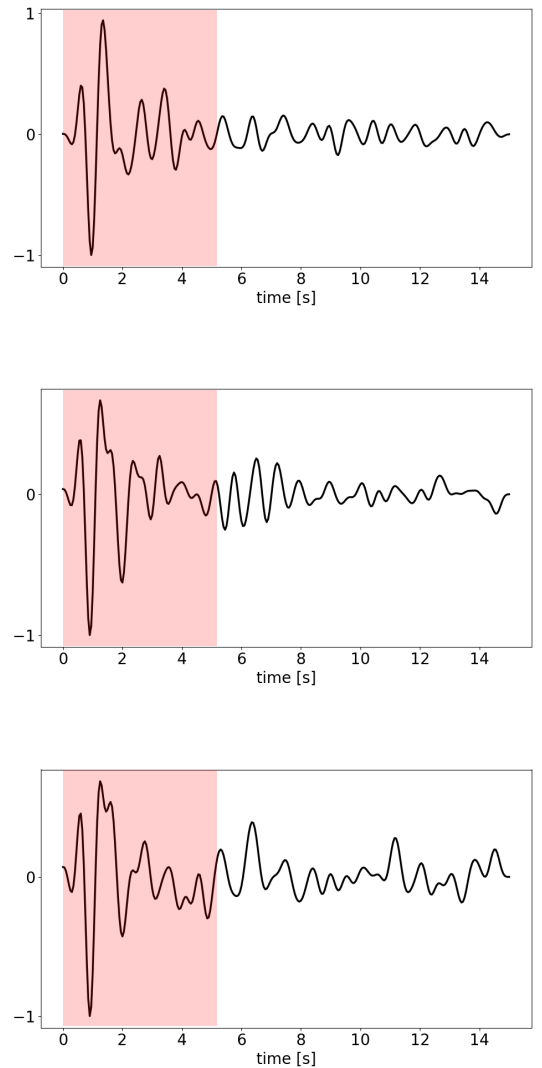

Figure 8: Comparison of $2 \mathrm{~Hz}$ synthetic (left) and observed (right) seismograms at the Yellowknife array (YKA, Canada) generated by USSR nuclear explosions in the Degelen Mountain range (67.0 degrees epicentral distance). The match of the pulse shapes in the highlighted time window is remarkable despite the lower corner frequency of the synthetics. The synthetics were generated with simple assumptions of an isotropic explosion source, a Gaussian moment rate function, a 1-D model, and NASA's SRTM topography (jpl.nasa.gov/srtm). With higher frequency modelling and more realistic source and model parameters, magnitude and yield estimates could be refined, and fitting waveform features could be used to constrain locations of numerical explosions. 
complex waveform features observed teleseismically. Sourceside modelling can also account for heterogeneous small-scale geologic structures, including random media, for realistic finite faults [22] or dynamic ruptures [27, 69], and for non-linear hydrodynamic interactions [71]. In all such applications, tradeoffs between various structural and source parameters - and how such interactions translate to teleseismic signals - could be evaluated. Moreover, applying backprojection [30, 72] or time-reversal [10] to synthetics could help to better map the time and space history of high-frequency body waves and their source-time evolution, highlighting interactions with specific scatterers in the source region.

\subsection{Depth}

Embedding 3-D solvers at depth and modelling localised smallscale structures along the propagation path could improve our understanding of teleseismic signatures - or lack thereof - of deep mantle structures and provide important constraints on the evolution of the Earth's interior. Global tomographic models are in reasonable agreement for large-scale 3-D structures $[4,5]$, but diverge at smaller length scales as a result of structural modelling assumptions and insufficient data coverage. A multi-scale view of thermal and chemical heterogeneities in the interior of the Earth, however, carries implications for global mantle convection and the Earth's heat budget, including coremantle exchanges and geochemical as well as mineralogical constraints $[21,28,58]$. Synthetic waveforms at high frequencies could provide insights into seismic properties of structures in the mid-mantle and around the CMB, such as velocities, densities, anisotropy and attenuation, as well as topography [e.g. 17] and sharpness of the lateral (e.g. D", 660 and Moho) and vertical (e.g. LLSVP, ULVZ) interfaces [e.g. 66]. Hybrid methods can also be directly adapted to localised tomographic studies based on full waveforms in order to improve the resolution of global Earth models at depth [39].

\subsection{Receiver side}

Finally, receiver-side hybrid modelling could provide a numerical means of exploring the ever larger high quality datasets from seismic arrays, such as USArray, AlpArray or Hi-net. Initially installed to improve the signal-to-noise ratio of teleseismic body waves from nuclear explosions, seismic arrays - with their regular and closely spaced instrument configurations - have since been used to study the Earth's fine-scale structure. Mirroring such dense datasets with high-frequency hybrid modelling would help not only in the interpretation of seismic recordings, but also in a systematic analysis of various array techniques, such as beamforming and its derivatives, different slant stacking techniques, and frequency-wave number analysis (for a review of array methods, see for example Rost and Thomas [56]). Receiver-side injection methods can also be used for explicit studies of study have also recently been used to study the effects of Moho and surface topography on teleseismic wavefields [44], to carry out parametric studies of inversion methods $[6,73]$, and to locally improve the resolution of tomographic models $[7,16,39]$

\section{Discussions}

Applying the principles of wavefield injection and wavefield extrapolation, we propose a global hybrid framework built around Instaseis databases that seeks a compromise between the availability of computational resources and simulating strong 3-D scattering of teleseismic body-waves at high frequencies. The methodology boasts an unprecedented level of flexibility, and can provide up to a 100,000 fold speed-up relative to full 3-D global solvers. With the databases serving as a once-and-for-all solution to wave propagation in spherically symmetric Earth models, the global wavefields are applicable to a variety of problems, shifting the heavy computational costs to long-term storage requirements. A single database can not only accommodate for different geometries and locations of the local domain within the background model, but also can be used for coupling with multiple local 3-D solvers of choice. Moreover, it allows alterations in source properties, such as locations, source-time functions and radiation patterns, leaving such choices to later hybrid simulations. With the ability to generate and store wavefields in the $1-4 \mathrm{~Hz}$ frequency range, and with the flexibility of the subsequent local modelling, the hybrid method can reach the highest frequencies observed teleseismically and opens global wave propagation to a new parameter space. The method has been applied to generate $2 \mathrm{~Hz}$ teleseismic seismograms bearing the signature of local 3-D topography of a nuclear explosions test site, successfully reproducing amplitudes and complex waveform features present in observed data [52, 53, in preparation]

Another novel method separating background and scattered wavefields has recently been proposed, entirely within the framework of AxiSEM3D [37], for high-frequency simulations with localised strong scatterers at depth. The method is precise for multiple, local interactions between a 3-D domain and a background wavefield, and allows for 3-D global models. It is, however, significantly more expensive for 3-D global simulations, and addresses a different suite of problems. Our method is geared towards higher-frequency applications, and it is complementary in its flexibility, its ease of use by relying on precomputed databases, and its plug-in approach for multiple solvers.

Two-step methods, however, are not suitable for problems where scattering is not localised. Not all wavefield interactions are accounted for with a one-way approach to coupling given the lack of a dynamic exchange of information on the boundary. All scattering effects are present within the local domain both for wavefield injection and extrapolation, and the scattered wavefield can leave the local domain. However, it can never return into the local domain in order to interact with embedded 3D structures again, as the global and local solvers do not act simultaneously or iteratively, but sequentially. In global seismology the effects of such higher order long range scattering at teleseismic distances are often small relative to primary interactions that the method can help understand. When designing a problem, however, it is of paramount importance to understand this limitation and the potential effect in can have on resulting waveforms.

Future developments of the framework include embedding multiple domains, including solid-fluid interfaces, and using 3-D background media if precomputed databases such as Instaseis for 3D models were available, which may be a future direction for machine-learning aided wave propagation [45]. The code for 
the framework is open source and is available as a modification of Instaseis at https://github.com/martapien/instaseis_hybrid.

\section{ACKNOWLEDGEMENTS}

We publish this work in memory of Dimitri Komatitsch, a dear colleague and friend who encouraged and provided support, and who facilitated collaboration that led to the completion of the project. We are grateful to Stephen Beller and Sébastien Chevrot who modified SPECFEM3D Cartesian for coupling with AxiSEM - the modifications were further adapted and formed the basis of the Instaseis coupling. MP also thanks Martin van Driel for AxiSEM and Instaseis support, and Stuart Nippress for help with the WPP coupling.

The work was funded by the European Union's Horizon 2020 research and innovation programme under the Marie Sklodowska-Curie grant agreement No 641943 and used the ARCHER UK National Supercomputing Service (http://www.archer.ac.uk).

This preprint is published under the CC BY 4.0 license (https://creativecommons.org/licenses/by/4.0/).

The file has been compiled with the latex template provided by https://github.com/brenhinkeller/preprint-template.tex.

\section{REFERENCES}

[1] K. Aki and P. G. Richards. Quantitative Seismology. University Science Books, Sausalito, CA, second edition edition, 2002.

[2] D. Al-Attar and J. H. Woodhouse. Calculation of seismic displacement fields in self-gravitating earth models-applications of minors vectors and symplectic structure. Geophys. J. Int., 175(3):1176-1208, 2008. ISSN 1365-246X. doi: 10.1111/j.1365-246X.2008.03961.x.

[3] Z. Alterman and F. Karal. Propagation of elastic waves in layered media by finite difference methods. Bull. Seismol. Soc. Am., 58(1):367-398, 1968.

[4] L. Auer, L. Boschi, T. W. Becker, T. Nissen-Meyer, and D. Giardini. Savani: A variable resolution whole-mantle model of anisotropic shear velocity variations based on multiple data sets. J. Geophys. Res. Solid Earth, 119 (4):3006-3034, 2014. ISSN 2169-9356. doi: 10.1002/ 2013JB010773.

[5] T. W. Becker and L. Boschi. A comparison of tomographic and geodynamic mantle models. Geochem. Geophys. Geosyst., 3(1), 2002. ISSN 15252027. doi: 10.1029/2001GC000168.

[6] S. Beller, V. Monteiller, L. Combe, S. Operto, and G. Nolet. On the sensitivity of teleseismic full-waveform inversion to earth parametrization, initial model and acquisition design. Geophys. J. Int., 212(2):1344-1368, 2018. ISSN 0956540X, 1365-246X. doi: 10.1093/gji/ggx480.

[7] S. Beller, V. Monteiller, S. Operto, G. Nolet, A. Paul, and L. Zhao. Lithospheric architecture of the South-Western Alps revealed by multiparameter teleseismic full-waveform inversion. Geophys. J. Int., 212(2):1369-1388, 2018. ISSN 0956-540X, 1365-246X. doi: 10.1093/gji/ggx216.

[8] J. Bielak. Domain Reduction Method for ThreeDimensional Earthquake Modeling in Localized Regions,
Part I: Theory. Bull. Seismol. Soc. Am., 93(2):817-824, 2003. ISSN 0037-1106. doi: 10.1785/0120010251.

[9] J. Bielak and P. Christiano. On the effective seismic input for non-linear soil-structure interaction systems. Earthq. Eng. Struct. Dyn., 12(1):107-119, 1984. ISSN 00988847, 10969845. doi: 10.1002/eqe.4290120108.

[10] L. Boschi, I. Molinari, and M. Reinwald. A simple method for earthquake location by surface-wave time reversal. Geophys. J. Int., 215(1):1-21, 2018. ISSN 0956-540X. doi: $10.1093 /$ gji/ggy 261 .

[11] F. Broggini, M. Vasmel, J. O. A. Robertsson, and D.-J. van Manen. Immersive boundary conditions: Theory, implementation, and examples. GEOPHYSICS, 82(3): T97-T110, 2017. ISSN 0016-8033, 1942-2156. doi: 10.1190/geo2016-0458.1.

[12] Y. Capdeville, E. Chaljub, and J. P. Montagner. Coupling the spectral element method with a modal solution for elastic wave propagation in global earth models. Geophys. J. Int., 152(1):34-67, 2003. ISSN 0956-540X, 1365-246X. doi: 10.1046/j.1365-246X.2003.01808.x.

[13] Y. Capdeville, A. To, and B. Romanowicz. Coupling spectral elements and modes in a spherical Earth: An extension to the 'sandwich' case. Geophys. J. Int., 154 (1):44-57, 2003. ISSN 0956540X, 1365246X. doi: 10.1046/j.1365-246X.2003.01959.x.

[14] E. Chaljub, D. Komatitsch, J.-P. Vilotte, Y. Capdeville, B. Valette, and G. Festa. Spectral-element analysis in seismology. Adv. Geophys., 48:365-419, 2007. doi: 10. 1016/S0065-2687(06)48007-9.

[15] S. Chevrot, N. Favier, and D. Komatitsch. Shear wave splitting in three-dimensional anisotropic media. Geophys. J. Int., 159(2):711-720, 2004. ISSN 0956540X, 1365246X. doi: 10.1111/j.1365-246X.2004.02432.x.

[16] P. Clouzet, Y. Masson, and B. Romanowicz. Box Tomography: First application to the imaging of upper-mantle shear velocity and radial anisotropy structure beneath the North American continent. Geophys. J. Int., 213(3): 1849-1875, 2018. ISSN 0956-540X, 1365-246X. doi: 10.1093/gji/ggy078.

[17] A. Colombi, T. Nissen-Meyer, L. Boschi, and D. Giardini. Seismic waveform inversion for core-mantle boundary topography. Geophys. J. Int., 198(1):55-71, 2014. ISSN 0956-540X. doi: 10.1093/gji/ggu112.

[18] P. Cupillard, E. Delavaud, G. Burgos, G. Festa, J.-P. Vilotte, Y. Capdeville, and J.-P. Montagner. RegSEM: A versatile code based on the spectral element method to compute seismic wave propagation at the regional scale. Geophys. J. Int., 188(3):1203-1220, 2012. ISSN 0956540X. doi: 10.1111/j.1365-246X.2011.05311.x.

[19] M. Dumbser and M. Käser. An arbitrary high-order discontinuous Galerkin method for elastic waves on unstructured meshes - II. The three-dimensional isotropic case. Geophys. J. Int., 167(1):319-336, 2006. ISSN 0956540X, 1365246X. doi: 10.1111/j.1365-246X.2006.03120.x.

[20] A. M. Dziewonski and B. Romanowicz. Deep Earth Seismology: An Introduction and Overview. In Treatise on Geophysics, volume 1. Elsevier, second edition edition, 2007. 
[21] A. M. Dziewonski, V. Lekic, and B. A. Romanowicz. Mantle Anchor Structure: An argument for bottom up tectonics. Earth Planet. Sci. Lett., 299(1):69-79, 2010. ISSN 0012821X. doi: 10.1016/j.eps1.2010.08.013.

[22] U. S. G. S. Earthquake Hazards Program. Finite Fault Database (ANSS ComCat). U.S. Geological Survey, Earthquake Hazards Program, 2017. doi: 10.5066/F7MS3QZH.

[23] M. Fink, C. Prada, F. Wu, and D. Cassereau. Self focusing in inhomogeneous media with time reversal acoustic mirrors. In IEEE Ultrasonics Symposium, pages 681-686, Montreal, Que., Canada, 1989. IEEE. doi: 10.1109/ULTSYM.1989.67072.

[24] J. T. Fokkema and P. M. van den Berg. Seismic Applications of Acoustic Reciprocity. Elsevier Science Publishers, Amsterdam, 1993.

[25] W. Friederich and J. Dalkolmo. Complete synthetic seismograms for a spherically symmetric earth by a numerical computation of the Green's function in the frequency domain. Geophys. J. Int., 122(2):537-550, 1995. ISSN 1365-246X. doi: 10.1111/j.1365-246X.1995.tb07012.x.

[26] M. Galis, P. Moczo, and J. Kristek. A 3-D hybrid finitedifference-finite-element viscoelastic modelling of seismic wave motion. Geophys. J. Int., 175(1):153-184, 2008. ISSN 0956540X, 1365246X. doi: 10.1111/j.1365-246X. 2008.03866.x.

[27] R. A. Harris, M. Barall, B. Aagaard, S. Ma, D. Roten, K. Olsen, B. Duan, D. Liu, B. Luo, K. Bai, J.-P. Ampuero, Y. Kaneko, A.-A. Gabriel, K. Duru, T. Ulrich, S. Wollherr, Z. Shi, E. Dunham, S. Bydlon, Z. Zhang, X. Chen, S. N. Somala, C. Pelties, J. Tago, V. M. Cruz-Atienza, J. Kozdon, E. Daub, K. Aslam, Y. Kase, K. Withers, and L. Dalguer. A Suite of Exercises for Verifying Dynamic Earthquake Rupture Codes. Seismol. Res. Lett., 89(3):1146-1162, 2018. ISSN 0895-0695. doi: 10.1785/0220170222.

[28] G. R. Helffrich and B. Wood. The Earth's Mantle. Nature, 412:501-7, 2001. doi: 10.1038/35087500.

[29] H. Igel, T. Nissen-Meyer, and G. Jahnke. Wave propagation in 3D spherical sections: Effects of subduction zones. Phys. Earth Planet. Inter., 132(1-3):219-234, 2002. ISSN 00319201. doi: 10.1016/S0031-9201(02)00053-5.

[30] M. Ishii, P. M. Shearer, H. Houston, and J. E. Vidale. Extent, duration and speed of the 2004 Sumatra-Andaman earthquake imaged by the Hi-Net array. Nature, 435 (7044):933-936, 2005. ISSN 1476-4687. doi: 10.1038/ nature 03675 .

[31] K. Kawai, N. Takeuchi, and R. J. Geller. Complete synthetic seismograms up to $2 \mathrm{~Hz}$ for transversely isotropic spherically symmetric media. Geophys. J. Int., 164(2):411424, 2006. ISSN 1365-246X. doi: 10.1111/j.1365-246X. 2005.02829.x.

[32] D. Komatitsch and J. Tromp. Spectral-element simulations of global seismic wave propagation-II. Three-dimensional models, oceans, rotation and self-gravitation. Geophys. J. Int., 150(1):303-318, 2002. ISSN 0956540X, 1365246X. doi: 10.1046/j.1365-246X.2002.01716.x.

[33] D. Komatitsch and J. Tromp. Spectral-element simulations of global seismic wave propagation-I. Validation. Geophys.
J. Int., 149(2):390-412, 2002. ISSN 0956540X, 1365246X. doi: 10.1046/j.1365-246X.2002.01653.x.

[34] M. Kristeková, J. Kristek, and P. Moczo. Time-frequency misfit and goodness-of-fit criteria for quantitative comparison of time signals. Geophys. J. Int., 178(2):813-825, 2009. ISSN 0956540X, 1365246X. doi: 10.1111/j.1365-246X. 2009.04177.x.

[35] K. Leng, T. Nissen-Meyer, and M. van Driel. Efficient global wave propagation adapted to 3-D structural complexity: A pseudospectral/spectral-element approach. Geophys. J. Int., 207(3):1700-1721, 2016. ISSN 0956-540X, 1365-246X. doi: 10.1093/gji/ggw363.

[36] K. Leng, T. Nissen-Meyer, M. van Driel, K. Hosseini, and D. Al-Attar. AxiSEM3D: Broad-band seismic wavefields in 3-D global earth models with undulating discontinuities. Geophys. J. Int., 217(3):2125-2146, 2019. ISSN 0956540X, 1365-246X. doi: 10.1093/gji/ggz092.

[37] K. Leng, J. Korenaga, and T. Nissen-Meyer. 3-D scattering of elastic waves by small-scale heterogeneities in the Earth's mantle. Geophys J Int, 223(1):502-525, 2020. ISSN 0956-540X. doi: 10.1093/gji/ggaa331.

[38] C. Lin, V. Monteiller, K. Wang, T. Liu, P. Tong, and Q. Liu. High-frequency seismic wave modelling of the deep Earth based on hybrid methods and spectral-element simulations: A conceptual study. Geophys J Int, 219(3):1948-1969, 2019. ISSN 0956-540X. doi: 10.1093/gji/ggz413.

[39] Y. Masson and B. Romanowicz. Fast computation of synthetic seismograms within a medium containing remote localized perturbations: A numerical solution to the scattering problem. Geophys. J. Int., 208(2):674-692, 2017. ISSN 0956-540X, 1365-246X. doi: 10.1093/gji/ggw412.

[40] Y. Masson and B. Romanowicz. Box tomography: Localized imaging of remote targets buried in an unknown medium, a step forward for understanding key structures in the deep Earth. Geophys. J. Int., 211(1):141-163, 2017. ISSN 0956-540X, 1365-246X. doi: 10.1093/gji/ggx141.

[41] Y. Masson, P. Cupillard, Y. Capdeville, and B. Romanowicz. On the numerical implementation of time-reversal mirrors for tomographic imaging. Geophys. J. Int., 196 (3):1580-1599, 2014. ISSN 1365-246X, 0956-540X. doi: $10.1093 / \mathrm{gji} / \mathrm{ggt} 459$.

[42] P. Moczo, E. Bystrick, J. Kristek, J. M. Carcione, and M. Bouchon. Hybrid Modeling of P-SV Seismic Motion at Inhomogeneous Viscoelastic Topographic Structures. Bull. Seismol. Soc. Am., 87(5):1305-1323, 1997.

[43] P. Moczo, J. Kristek, and M. Galis. The Finite-Difference Modelling of Earthquake Motions: Waves and Ruptures. Cambridge University Press, Cambridge, UK, first edition edition, 2014.

[44] V. Monteiller, S. Chevrot, D. Komatitsch, and N. Fuji. A hybrid method to compute short-period synthetic seismograms of teleseismic body waves in a 3-D regional model. Geophys. J. Int., 192(1):230-247, 2013. ISSN 1365-246X, 0956-540X. doi: 10.1093/gji/ggs006.

[45] B. Moseley, A. Markham, and T. Nissen-Meyer. Fast approximate simulation of seismic waves with deep learning. arXiv preprint arXiv:1807.06873. 2018. 
[46] S. Nilsson, N. A. Petersson, B. Sjogreen, and H.-O. Kreiss. Stable difference approximations for the elastic wave equation in second order formulation. SIAM J. Numer. Anal., 45:1902-1936, 2007.

[47] T. Nissen-Meyer, F. A. Dahlen, and A. Fournier. Sphericalearth Fréchet sensitivity kernels. Geophys. J. Int., 168 (3):1051-1066, 2007. ISSN 0956540X, 1365246X. doi: 10.1111/j.1365-246X.2006.03123.x.

[48] T. Nissen-Meyer, M. van Driel, S. C. Stähler, K. Hosseini, S. Hempel, L. Auer, A. Colombi, and A. Fournier. AxiSEM: Broadband 3-D seismic wavefields in axisymmetric media. Solid Earth, 5:425-445, 2014. ISSN 18699529. doi: 10.5194/se-5-425-2014.

[49] I. Opršal and J. Zahradnik. Three-dimensional finite difference method and hybrid modeling of earthquake ground motion. J. Geophys. Res., 107(B8):ESE2:1-16, 2002. ISSN 0148-0227. doi: 10.1029/2000JB000082.

[50] I. Opršal, C. Matyska, and K. Irikura. The source-box wave propagation hybrid methods: General formulation and implementation. Geophys. J. Int., 176(2):555-564, 2009. ISSN 0956540X, 1365246X. doi: 10.1111/j.1365-246X. 2008.03986.x.

[51] N. A. Petersson and B. Sjogreen. User's Guide to WPP Version 2.2. Lawrence Livermore National Laboratory, Livermore, CA, 2013.

[52] M. Pienkowska, S. Nippress, T. Nissen-Meyer, and D. Bowers. Evaluation of near-source scattering on explosion P waves at the Degelen test site, Part I: A manifestation of source complexity in observed waveforms. Manuscript in preparation. 2020.

[53] M. Pienkowska, S. Nippress, T. Nissen-Meyer, and D. Bowers. Evaluation of near-source scattering on explosion P waves at the Degelen test site, Part II: Modelling the effects of topography at teleseismic distances. Manuscript in preparation. 2020.

[54] J. O. A. Robertsson and C. H. Chapman. An efficient method for calculating finite-difference seismograms after model alterations. GEOPHYSICS, 65(3):907-918, 2000.

[55] S. Roecker, B. Baker, and J. McLaughlin. A finitedifference algorithm for full waveform teleseismic tomography. Geophys. J. Int., 181(2):1017-1040, 2010. ISSN 1365-246X. doi: 10.1111/j.1365-246X.2010.04553.x.

[56] S. Rost and C. Thomas. Array Seismology: Methods and Applications. Rev. Geophys., 40(3):2:1-27, 2002. ISSN 1944-9208. doi: 10.1029/2000RG000100.

[57] R. Stacey. Improved transparent boundary formulations for the elastic-wave equation. Bull. Seismol. Soc. Am., 78 (6):2089-2097, 1988. ISSN 0037-1106.

[58] B. Steinberger and T. H. Torsvik. A geodynamic model of plumes from the margins of Large Low Shear Velocity Provinces. Geochem. Geophys. Geosyst., 13(1), 2012. ISSN 1525-2027. doi: 10.1029/2011GC003808.

[59] P. Tong, C.-w. Chen, D. Komatitsch, P. Basini, and Q. Liu. High-resolution seismic array imaging based on an SEMFK hybrid method. Geophys. J. Int., 197(1):369-395, 2014. ISSN 1365-246X, 0956-540X. doi: 10.1093/gji/ggt508.
[60] S. Tsuboi, K. Ando, T. Miyoshi, D. Peter, D. Komatitsch, and J. Tromp. A 1.8 trillion degrees-of-freedom, 1.24 petaflops global seismic wave simulation on the K computer. The International Journal of High Performance Computing Applications, 30(4):411-422, 2016. ISSN 1094-3420, 1741-2846. doi: 10.1177/1094342016632596.

[61] M. van Driel and T. Nissen-Meyer. Optimized viscoelastic wave propagation for weakly dissipative media. Geophys. J. Int., 199(2):1078-1093, 2014. ISSN 0956-540X. doi: $10.1093 / g j i / g g u 314$.

[62] M. van Driel and T. Nissen-Meyer. Seismic wave propagation in fully anisotropic axisymmetric media. Geophys. J. Int., 199(2):880-893, 2014. ISSN 0956-540X, 1365-246X. doi: $10.1093 /$ gji/ggu269.

[63] M. van Driel, L. Krischer, S. C. Stähler, K. Hosseini, and T. Nissen-Meyer. Instaseis: Instant global seismograms based on a broadband waveform database. Solid Earth, 6(2):701-717, 2015. ISSN 1869-9529. doi: 10.5194/ se-6-701-2015.

[64] M. van Driel, C. Boehm, L. Krischer, and M. Afanasiev. Accelerating numerical wave propagation by wavefieldadapted meshes, part 1: Forward and adjoint modelling. Geophys. J. Int., 2019.

[65] D.-J. van Manen, J. O. A. Robertsson, and A. Curtis. Exact wave field simulation for finite-volume scattering problems. J. Acoust. Soc. Am., 122(4):EL115-EL121, 2007. ISSN 0001-4966. doi: 10.1121/1.2771371.

[66] E. A. Vanacore, S. Rost, and M. S. Thorne. Ultralowvelocity zone geometries resolved by multidimensional waveform modelling. Geophys. J. Int., 206(1):659-674, 2016. ISSN 0956-540X. doi: 10.1093/gji/ggw114.

[67] L. Wen and D. V. Helmberger. A two-dimensional P-SV hybrid method and its application to modeling localised structures near the core-mantle boundary. J. Geophys. Res., 103(B8):17901-17918, 1998.

[68] B. Willemsen, A. Malcolm, and W. Lewis. A numerically exact local solver applied to salt boundary inversion in seismic full-waveform inversion. Geophys. J. Int., 204 (3):1703-1720, 2016. ISSN 0956-540X, 1365-246X. doi: $10.1093 / g j i / g g v 547$.

[69] S. Wollherr, A.-A. Gabriel, and C. Uphoff. Off-fault plasticity in three-dimensional dynamic rupture simulations using a modal Discontinuous Galerkin method on unstructured meshes: Implementation, verification and application. Geophys. J. Int., 214(3):1556-1584, 2018. ISSN 0956-540X, 1365-246X. doi: 10.1093/gji/ggy213.

[70] W. Wu, S. Ni, Z. Zhan, and S. Wei. An SEM-DSM three-dimensional hybrid method for modelling teleseismic waves with complicated source-side structures. Geophys. J. Int., 215(1):133-154, 2018. ISSN 0956-540X, 1365-246X. doi: 10.1093/gji/ggy273.

[71] H. Xu, A. Rodgers, I. Lomov, N. Petersson, B. Sjogreen, and O. Vorobiev. Simulation of Explosion Ground Motions Using a Hydrodynamic-to-Elastic Coupling Approach in Three Dimensions. The Bulletin of the Seismological Society of America, 103:1629-1639, 2013. doi: $10.1785 / 0120120180$. 
[72] Y. Xu, K. D. Koper, O. Sufri, L. Zhu, and A. R. Hutko. Rupture imaging of the Mw 7.9 12 May 2008 Wenchuan earthquake from back projection of teleseismic $\mathrm{P}$ waves. Geochem. Geophys. Geosyst., 10(4), 2009. ISSN 15252027. doi: 10.1029/2008GC002335.

[73] S. Yuan, N. Fuji, S. Singh, and D. Borisov. Localized timelapse elastic waveform inversion using wavefield injection and extrapolation: 2-D parametric studies. Geophys. J. Int., 209(3):1699-1717, 2017. ISSN 0956-540X, 1365-246X. doi: $10.1093 / g j i / g g x 118$.

[74] L. Zhao, L. Wen, L. Chen, and T. Zheng. A twodimensional hybrid method for modeling seismic wave propagation in anisotropic media. J. Geophys. Res., 113:B12307, 2008. ISSN 0148-0227. doi: 10.1029/ 2008JB005733. 